\title{
Medidas antropométricas em idosos assistidos na atenção básica e sua associação com gênero, idade e síndrome da fragilidade: dados do EMI-SUS
}

\author{
Anthropometric measurements in elderly assisted in primary health care \\ and their association with gender, age and frailty syndrome: EMI-SUS data
}

\author{
Vera Elizabeth Closs ${ }^{1}$, Laura Schlatter Rosemberg' ${ }^{1}$ Betina da Gama Ettrich ${ }^{2}$, Irenio Gomes ${ }^{1}$, \\ Carla Helena Augustin Schwanke ${ }^{1} \bowtie$ \\ 1 Programa de Pós-graduação em Gerontologia Biomédica do Instituto de Geriatria e Gerontologia da Pontifícia Universidade Católica do \\ Rio Grande do Sul (IGG-PUCRS). Porto Alegre, RS. \\ 2 Programa de Pós-Graduação em Ciências da Saúde: Cardiologia e Ciências Cardiovasculares da Universidade Federal do \\ Rio Grande do Sul (UFRGS). Porto Alegre, RS.
}

Estudo apoiado pela Fundação de Amparo à Pesquisa do Estado do Rio Grande do Sul (FAPERGS). V. E. C. recebeu bolsa de estudos da Coordenação de Aperfeiçoamento de Pessoal de Nível Superior (CAPES).

\section{RESUMO}

Objetivos: Descrever medidas antropométricas em idosos assistidos na atenção básica e analisar sua associação com gênero, idade e síndrome da fragilidade.

Métodos: Este estudo transversal é parte do Estudo Epidemiológico e Clínico dos Idosos Atendidos pela Estratégia Saúde da Família de Porto Alegre-RS-Brasil (EMI-SUS). Foram obtidas dos idosos 27 medidas antropométricas (analisadas de forma isolada ou em equações, totalizando 41 dados de medidas antropométricas) e dados para determinação da síndrome de fragilidade (Fenótipo de Fried). Foram utilizados o teste de Kolmogorov-Smirnov, o teste t de Student e o teste de análise de variância (ANOVA). Considerou-se o nível de confiança de $95 \%$. Resultados: A amostra incluiu 583 idosos, sendo 371 (63,6\%) mulheres. Homens apresentaram médias significativamente superiores de peso, estatura, altura do joelho, circunferências (antebraço, muscular do braço, pescoço, punho), área muscular do braço, relações (cintura/ quadril, cintura/coxa, dobras tronco/extremidades) e índices (sagital, de conicidade). Mulheres apresentaram médias superiores do índice de massa corporal, circunferências (braço, cintura, coxa, quadril), dobras cutâneas (bicipital, tricipital, subescapular, suprailíaca, peitoral, coxa, panturrilha, média seis dobras), área de gordura do braço, relação cintura/estatura e relação diâmetro abdominal sagital/estatura. Idosos mais jovens apresentaram medidas superiores das circunferências do antebraço, braço, músculo do braço, coxa e área muscular do braço em relação aos mais velhos. Idosos robustos tinham estatura, altura do joelho, circunferência muscular do braço e área muscular do braço superiores e área de gordura do braço, dobras cutâneas da coxa, peitoral, bicipital, tricipital, panturrilha e relação diâmetro abdominal sagital/estatura inferiores aos frágeis e pré-frágeis. Idosos frágeis tinham circunferências da panturrilha e antebraço menores e relações cintura/estatura, cintura/coxa, cintura/panturrilha e índices de conicidade e sagital, maiores que os robustos.

Conclusões: As medidas antropométricas mostraram-se associadas com gênero, idade e fragilidade. Nos homens, as médias das medidas de tamanho, compleição e de reserva de massa muscular foram maiores. Nas mulheres, as médias das medidas de reserva adiposa foram maiores e a associação das medidas com a faixa etária foi mais frequente. Fragilidade mostrou-se associada com perda de massa muscular. Idosos frágeis apresentaram medidas inferiores de tamanho e compleição.

DESCRITORES: antropometria; idoso fragilizado; atenção primária à saúde.

\section{ABSTRACT}

Aims: To describe anthropometric measurements and analyze their association with gender, age, and frailty syndrome in elderly assisted in primary health care.

Methods: This cross-sectional study is part of the Multidimensional Study of the Elderly in the Family Health Strategy in Porto Alegre, RS, Brazil (EMI-SUS). Twenty-seven anthropometric measurements (analyzed in isolation or in equations, totaling 41 measurements) and data for determining the frailty (Fried Phenotype) were collected from elderly individuals. Kolmogorov-Smirnov test, Student t test and analysis of variance (ANOVA) were used for statistical analysis, considering a confidence level of $95 \%$.

Results: The sample included 583 elderly, of whom 371 (63.6\%) were women. Men presented significantly higher means for weight, height, knee-height; forearm, muscle arm, neck and wrist circumferences; muscle arm-area; ratios for waist-to-hip, waist-to-thigh, and trunk-to-skinfolds-extremities; and sagittal and conicity indexes. Women showed higher means for body mass index; arm, waist, thigh and hip circumferences; biceps, triceps, subscapular, suprailiac, pectoral, thigh, calf, and mean of six skinfolds; fat arm area, waist-to-height ratio and sagittal abdominal diameter-to-height ratio. The younger elderly presented higher measures of forearm, arm, muscle arm and thigh circumferences; and muscle arm area, in relation to the older. The robust elderly had higher measures of height, knee-height, muscle arm 
circumference and muscle arm area; and lower fat arm area, thigh, pectoral, biceps, triceps and calf skinfolds; and sagittal abdominal diameterto-height ratio, than the pre-frail and frail seniors. The frail elderly had lower calf and forearm circumferences than the robust elderly, and higher waist-to-height, waist-to-thigh and waist-to-calf ratios, and conicity and sagittal indexes.

Conclusions: Anthropometric measures were associated with gender, age and frailty. In men, the mean measures of size, build and muscle mass reserve were higher. In women, the mean measures of fat reserve were higher and the association of the measures with age group was more frequent. Frailty proved to be associated with muscle mass loss. The frail elderly had lower measures of size and complexion.

KEY WORDS: anthropometry; frail elderly; primary health care.

\begin{abstract}
Abreviaturas: $\mathrm{AGB}$, área de gordura do braço; $\mathrm{AMBc}$, área muscular do braço corrigida; $\mathrm{CAB}$, circunferência do antebraço; $\mathrm{CB}$, circunferência do braço; $\mathrm{CC}$, circunferência da cintura; $\mathrm{CMB}$, circunferência muscular do braço; $\mathrm{CP}$, circunferência da panturrilha; CQ, circunferência do quadril; DAS, diâmetro abdominal sagital; DCA, dobra cutânea abdominal; DCT, dobra cutânea tricipital; EMI-SUS, Estudo Epidemiológico e Clínico dos Idosos Atendidos pela Estratégia Saúde da Família de Porto Alegre, Brasil; IMC, índice de massa corporal; RCE, relação cintura/estatura.
\end{abstract}

\section{INTRODUÇÃO}

Medidas antropométricas têm sido muito úteis, ao longo dos anos, na avaliação nutricional de indivíduos, principalmente quando recursos mais sofisticados e onerosos não estão disponíveis [1]. As dimensões corporais refletem a saúde geral e o bemestar de indivíduos e populações e, podem, ainda, ser utilizadas para determinar vulnerabilidade ou sobrevivência [2].

O processo do envelhecimento está relacionado a significativas mudanças na composição corporal [3] que ocorrem de forma diferente em homens e mulheres nas suas diversas fases da vida, influenciando a antropometria [1]. Essas alterações são mudanças anatômicas progressivas que repercutem nas condições de saúde dos indivíduos e têm sido associadas com baixo desempenho físico, inatividade, velocidade de marcha reduzida e diminuição da mobilidade, características comuns da síndrome da fragilidade $[3,4]$.

A síndrome da fragilidade apresenta diferentes estágios de gravidade, com uma relação dinâmica entre as suas fases [4,5], e tem sido associada com risco aumentado de acúmulo de déficits e de mortalidade por todas as causas, na população idosa [6,7]. No desenvolvimento de fragilidade, vários processos fisiopatológicos estão envolvidos e, entre eles, observase uma estreita relação da fragilidade com perda de peso, sarcopenia, obesidade, aspectos nutricionais e de composição corporal [8]. Ambos, baixo peso e obesidade, estão associados a limitações funcionais. Além disso, a perda de massa magra nem sempre é acompanhada de perda de massa gorda, podendo, muitas vezes, ocorrer aumento do tecido adiposo, caracterizando-se a obesidade sarcopênica [9].

Estima-se que de 10 a $25 \%$ dos idosos com mais de 65 anos e $46 \%$ dos acima de 85 anos, que vivem na comunidade, sejam frágeis. Na Estratégia Saúde da Família, o reconhecimento dos possíveis sinais referentes à síndrome é fundamental [10]. Nesse contexto, a antropometria apresenta-se como um método útil, não invasivo, rápido, de aplicação relativamente simples, de baixo custo [11], uma técnica portátil e universalmente aplicável [12] na avaliação do estado nutricional, da sarcopenia, do declínio funcional e de condições crônicas de saúde, fatores envolvidos na fisiopatologia da fragilidade $[8,13,14]$.

A antropometria é relativamente pouco utilizada nos indivíduos mais velhos. Dificuldades na obtenção de algumas medidas, atribuídas às alterações fisiológicas que ocorrem com o envelhecimento [15], têm limitado os registros quantitativos destas modificações. A avaliação nutricional de idosos, utilizando a antropometria, exige referências antropométricas ou valores usuais para comparação [16]. Na literatura científica relacionada à área de geriatria e gerontologia existe uma carência de estudos populacionais com dados e informações aprofundadas sobre parâmetros antropométricos de idosos [2], e a Organização Mundial da Saúde [11] tem enfatizado a necessidade de estudos que possam trazer informações referentes a populações específicas, como é o caso dos idosos.

Assim, este estudo teve como principal objetivo descrever medidas antropométricas de idosos assistidos na Estratégia Saúde da Família de Porto Alegre, Rio Grande do Sul, e analisar sua associação com gênero, idade e síndrome da fragilidade.

\section{MÉTODOS}

Foi realizado um estudo transversal, parte integrante do Estudo Epidemiológico e Clínico dos Idosos Atendidos pela Estratégia Saúde da Família (ESF) do Município de Porto Alegre, Rio Grande do Sul 
(EMI-SUS), cuja metodologia encontra-se descrita em Gomes et al. [17]. Foram incluídos na amostra indivíduos idosos (com 60 anos ou mais) dos quais foram obtidos os registros antropométricos e os dados necessários para a determinação da síndrome da fragilidade (Fenótipo de Fried et al.) [4]. O EMI-SUS foi aprovado pelos Comitês de Ética em Pesquisa da Pontifícia Universidade Católica do Rio Grande do Sul (PUCRS) (protocolo no CEP-10/04967) e da Secretaria Municipal de Saúde de Porto Alegre (protocolo no 001.021434.10.7). O Termo de Consentimento Livre e Esclarecido foi obtido de todos os participantes.

Os dados foram coletados entre março de 2011 e dezembro de 2012. Inicialmente, agentes comunitários de saúde aplicaram um questionário geral na residência dos idosos para a obtenção dos dados sociodemográficos e de saúde. Posteriormente, uma equipe composta por três nutricionistas treinadas e certificadas pela International Society for the Advancement of Kinanthropometry (ISAK) [18] coletou as medidas antropométricas e os dados necessários para determinação do fenótipo de fragilidade. Para essa fase, aos sábados, 36 idosos (18 em cada turno) eram buscados na comunidade e conduzidos a um ambulatório do Hospital São Lucas da PUCRS (HSLPUCRS), que, por questões logísticas, serviu de base para a coleta dos dados. As medidas antropométricas foram realizadas todas no mesmo dia para cada indivíduo, no lado direito do corpo. As circunferências foram obtidas com fita métrica graduada, flexível e inelástica e as dobras cutâneas com compasso Cescorf. As variáveis investigadas, bem como os instrumentos e os critérios diagnósticos utilizados neste estudo estão descritos no Quadro 1.

Quadro 1. Descrição dos instrumentos utilizados para a coleta das medidas antropométricas e do critério diagnóstico da síndrome da fragilidade.

\begin{tabular}{|c|c|}
\hline Medidas antropométricas & Instrumento/Critério diagnóstico \\
\hline Peso & Medida (kg) na balança Filizola com idoso sem sapatos e descartadas vestes e objetos pesados [19] \\
\hline Estatura & Medida $(\mathrm{m})$ com o estadiômetro acoplado à balança Filizola: idoso ereto [19] \\
\hline Índice de massa corporal & Índice de Quetelet = Peso (kg)/Estatura $(\mathrm{m})^{2}[20]$ \\
\hline Altura do Joelho & Medida $(\mathrm{cm})$ da distância entre a sola do pé e a superfície anterior da coxa, com idoso sentado [21] \\
\hline \multicolumn{2}{|l|}{ Circunferência } \\
\hline Antebraço & Medida (cm) na máxima circunferência do antebraço, perpendicularmente ao seu eixo longitudinal [18] \\
\hline Braço & Medida $(\mathrm{cm})$ no ponto médio entre o acrômio e o olecrano [19] \\
\hline Muscular do braço & Circunferência muscular do braço $(\mathrm{cm})=$ Circunferência braço $(\mathrm{cm})-[\pi \times($ Dobra cutânea tricipital $(\mathrm{mm}) / 10)]$ [22] \\
\hline Cintura no nível umbilical & Medida $(\mathrm{cm})$ no nível da cicatriz umbilical [1] \\
\hline Cintura menor & Medida $(\mathrm{cm})$ no ponto mais estreito do tronco [23] \\
\hline Cintura no ponto médio & Medida $(\mathrm{cm})$ no ponto médio entre a última costela e a crista ilíaca [24] \\
\hline Coxa & Medida $(\mathrm{cm})$ no ponto mesofemural [18] \\
\hline Panturrilha & Medida $(\mathrm{cm})$ horizontalmente na área de maior diâmetro da panturrilha, com idoso sentado [11] \\
\hline Pescoço & Medida $(\mathrm{cm})$ perpendicularmente ao eixo longo do pescoço, na menor circunferência acima da proeminência laríngea [19] \\
\hline Punho & Medida $(\mathrm{cm})$ no perímetro mínimo, com braço levemente flexionado [18] \\
\hline Quadril & Medida $(\mathrm{cm})$ no plano horizontal, no nível da extensão máxima dos glúteos. \\
\hline \multicolumn{2}{|l|}{ Dobra cutânea } \\
\hline Abdominal & Medida $(\mathrm{mm})$ aferida paralelamente ao eixo longitudinal do corpo, a $2 \mathrm{~cm}$ à direita da borda lateral da cicatriz umbilical [18] \\
\hline Bicipital & Medida $(\mathrm{mm})$ no ponto mesoumeral da face anterior do braço [18] \\
\hline Coxa & $\begin{array}{l}\text { Medida }(\mathrm{mm}) \text { paralela ao eixo longitudinal da coxa, sobre o músculo reto femoral, no ponto médio entre o ligamento } \\
\text { inguinal e a borda superior da patela [19] }\end{array}$ \\
\hline Panturrilha & $\begin{array}{l}\text { Medida }(\mathrm{mm}) \text { tomada no sentido paralelo ao eixo longitudinal da perna, na altura da sua maior circunferência, com o idoso } \\
\text { sentado [18] }\end{array}$ \\
\hline Peitoral & Medida (mm), pinçada no sentido diagonal, no ponto médio entre a linha axilar anterior e o mamilo $[23,25]$ \\
\hline Subescapular & $\begin{array}{l}\text { Medida }(\mathrm{mm}) \text { coletada a dois } \mathrm{cm} \text { abaixo do ângulo inferior da escápula, pinçada obliquamente a partir da referência } \\
\text { anatômica, seguindo na referência dos arcos costais }[26,27]\end{array}$ \\
\hline Suprailíaca & Medida (mm) aferida paralelamente ao eixo longitudinal do corpo, logo acima da crista ilíaca, segundo a linha média axilar [18] \\
\hline Tricipital & $\begin{array}{l}\text { Medida }(\mathrm{mm}) \text {, na face posterior do braço, paralelamente ao seu eixo longitudinal, no ponto médio entre a borda súperolateral } \\
\text { do acrômio e o olecrano }[19,28]\end{array}$ \\
\hline Média de dobras cutâneas & Dobras cutâneas $(\mathrm{mm})$ (abdominal + bicipital + panturrilha + subescapular + suprailíaca + tricipital)/6 [29] \\
\hline
\end{tabular}


Quadro 1. (Conclusão)

\begin{tabular}{|c|c|c|}
\hline Medidas antropométricas & \multicolumn{2}{|c|}{ Instrumento/Critério diagnóstico } \\
\hline Diâmetro Abdominal Sagital & \multicolumn{2}{|c|}{$\begin{array}{l}\text { Medida }(\mathrm{cm}) \text { da distância entre a superfície das costas e o topo do abdômen, aferida com Holtain-Kahn Caliper, com o idoso } \\
\text { deitado; tomada em seis pontos: na menor cintura entre o tórax e o quadril [30]; no nível umbilical [31]; no ponto médio } \\
\text { entre a borda superior da crista ilíaca e a borda inferior do rebordo costal; no nível das cristas ilíacas, entre a L4 e L5 [32]; } \\
\text { e na maior proeminência do abdômen, com o idoso em ortostatismo. }\end{array}$} \\
\hline \multicolumn{3}{|l|}{ Área } \\
\hline Gordura do braço & \multicolumn{2}{|c|}{$\begin{aligned} \text { Área de gordura do braço }\left(\mathrm{cm}^{2}\right)= & \{\text { [Circunferência do braço } \times(\text { Dobra cutânea tricipital } / 10)] / 2\}- \\
& \left.\left\{\pi \times(\text { Dobra cutânea tricipital } / 10)^{2}\right] / 4\right\}[33]\end{aligned}$} \\
\hline Muscular do braço corrigida & \multicolumn{2}{|c|}{$\begin{array}{l}\text { Área muscular do braço corrigida }\left(\mathrm{cm}^{2}\right): \\
\text { Homens }=\left\{\left[(\text { Circunferência do braço }-\pi \times \text { Dobra cutânea tricipital } / 10)^{2}\right] / 4 \pi\right\}-10 \\
\text { Mulheres }=\left\{\left[(\text { Circunferência do braço }-\pi \times \text { Dobra cutânea tricipital } / 10)^{2}\right] / 4 \pi\right\}-6,5[34]\end{array}$} \\
\hline \multicolumn{3}{|l|}{ Relação } \\
\hline Cintura/quadril & \multicolumn{2}{|c|}{ Circunferência da cintura $(\mathrm{cm})$ / circunferência do quadril $(\mathrm{cm})$ [11] } \\
\hline Cintura/estatura & \multicolumn{2}{|c|}{ Circunferência da cintura $(\mathrm{cm}) /$ estatura $(\mathrm{cm})[11]$} \\
\hline Cintura/coxa & \multicolumn{2}{|c|}{ Circunferência da cintura $(\mathrm{cm}) /$ circunferência da coxa $(\mathrm{cm})$ [35] } \\
\hline Cintura/panturrilha & \multicolumn{2}{|c|}{ Circunferência da cintura $(\mathrm{cm})$ / circunferência da panturrilha $(\mathrm{cm})$} \\
\hline Cintura/quadril/estatura & \multicolumn{2}{|c|}{ Quociente entre circunferência da cintura (cm) / circunferência do quadril (cm), e estatura (cm) [36] } \\
\hline DAS/estatura & \multicolumn{2}{|c|}{ Diâmetro abdominal sagital (cm) / estatura (cm) [36] } \\
\hline $\begin{array}{l}\text { Dobras cutâneas do } \\
\text { tronco/extremidades }\end{array}$ & \multicolumn{2}{|c|}{ Dobras cutâneas (subescapular + suprailíaca) / dobras cutâneas tricipital + panturrilha) [37] } \\
\hline \multicolumn{3}{|l|}{ Índice } \\
\hline Sagital & \multicolumn{2}{|c|}{ Diâmetro abdominal sagital (cm) / circunferência da coxa (cm) [35] } \\
\hline Conicidade & \multicolumn{2}{|c|}{ Índice $C=\frac{\text { Circunferência da Cintura }(\mathrm{m})}{\sqrt{\frac{\text { Peso corporal }(\mathrm{kg})}{\text { Estatura }(\mathrm{m})}}}$} \\
\hline \multicolumn{3}{|l|}{ Fragilidade } \\
\hline Fenótipo de Fried & \multicolumn{2}{|c|}{ Frágil: $\geq 3$ critérios presentes; Pré-frágil: 1 ou 2 critérios presentes; Robusto: nenhum critério presente [4] } \\
\hline Perda de peso & \multicolumn{2}{|c|}{ Autorrelato de perda de >4.55kg não intencional nos últimos 12 meses [4] } \\
\hline Fraqueza muscular & $\begin{array}{ll}\text { Preensão palmar }(\mathrm{kgf}) \text { medida com dinamôn } \\
\text { Homens } & \\
\text { IMC } \leq 24 & \text { Preensão palmar } \leq 29 \\
\text { IMC } 24.1-26 & \text { Preensão palmar } \leq 30 \\
\text { IMC } 26.1-28 & \text { Preensão palmar } \leq 30 \\
\text { IMC }>28 & \text { Preensão palmar } \leq 32\end{array}$ & 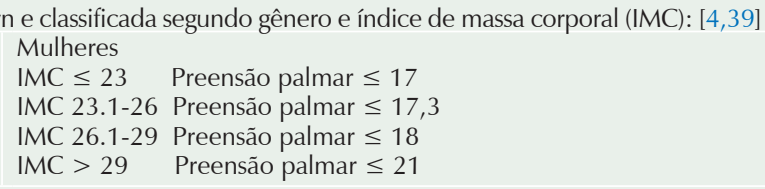 \\
\hline Exaustão & \multicolumn{2}{|c|}{$\begin{array}{l}\text { Autorrelato de fadiga, identificada por duas perguntas da versão brasileira da escala de depressão do Center for Epidemiologic } \\
\text { Studies (CES-D) [40] } \\
\text { Resposta " } 2 \text { " ou " } 3 \text { " para qualquer uma das questões [4] }\end{array}$} \\
\hline Baixa velocidade de marcha & $\begin{array}{l}\text { Caminhada ao longo de } 4,6 \mathrm{~m} \text {, classificada } \\
\geq 7 \text { segundos para: } \\
\text { Homens - Altura } \leq 1,73 \mathrm{~m} \\
\text { Mulheres - Altura } \leq 1,59 \mathrm{~m}\end{array}$ & $\begin{array}{l}\text { ênero e estatura: } \\
\geq 6 \text { segundos para: } \\
\text { Homens - Altura }>1,73 \mathrm{~m} \\
\text { Mulheres - Altura }>1,59 \mathrm{~m}[4]\end{array}$ \\
\hline Baixo nível de atividade física & \multicolumn{2}{|c|}{$\begin{array}{l}\text { Minnesota Leisure Time Activity Questionnaire [41]: } \\
<383 \text { kcal para homens e }<270 \text { kcal para mulheres [4,41] }\end{array}$} \\
\hline
\end{tabular}

As medidas antropométricas foram realizadas todas no mesmo dia para cada indivíduo, no lado direito do corpo. As circunferências foram obtidas com fita métrica graduada, flexível e inelástica e as dobras cutâneas, com compasso Cescorf; DAS: diâmetro abdominal sagital; $\pi: 3,14 ;$ IMC: Índice de massa corporal,

Fonte: os autores.

A análise estatística foi feita por meio do SPSS Statistics versão 17.0. O teste de Kolmogorov-Smirnov foi usado para verificar a normalidade da distribuição dos dados. A associação das variáveis antropométricas com gênero foi realizada através do teste $t$ de Student. Para idade (faixas etárias) e categorias de fragilidade utilizou-se o teste de análise de variância (ANOVA) com post hoc de Bonferroni. As análises foram realizadas considerando-se o nível de confiança de 95\% $(\alpha=5 \%)$.

\section{RESULTADOS}

A amostra analisada foi composta de 583 idosos. A média da idade foi de $68,5 \pm 7,1$ anos (intervalo de 60 a 103 anos). Os homens tinham média de idade de $67,7 \pm 6,4$ anos e as mulheres de $68,9 \pm 7,4$ anos. $\mathrm{Na}$ amostra total, o grupo etário com maior número de indivíduos foi de $60-70$ anos $(69,1 \%)$, seguido do grupo de $71-80$ anos $(24,4 \%)$. 
A maioria da amostra era do gênero feminino $(\mathrm{N}=371,63,6 \%)$ e a comparação entre os gêneros mostrou diferença significativa em 29 das 41 medidas avaliadas. Homens $(\mathrm{N}=212,36,4 \%)$ apresentaram médias superiores de peso, estatura, altura do joelho, circunferência do antebraço $(\mathrm{CAB})$, circunferência muscular do braço (CMB), circunferência do pescoço, circunferência do punho, área muscular do braço corrigida $(\mathrm{AMBc})$, relação cintura/quadril, relação cintura/coxa, relação dobras cutâneas do tronco/dobras cutâneas das extremidades e dos índices sagital e de conicidade (Tabela 1).

Mulheres tinham médias superiores de índice de massa corporal (IMC), circunferência do braço (CB), circunferência da cintura (CC) no nível umbilical, circunferência da coxa, circunferência do quadril (CQ), dobras cutâneas bicipital, tricipital (DCT), subescapular, suprailíaca, peitoral, da coxa, da panturrilha e média de seis dobras cutâneas, área de gordura do braço (AGB), relação circunferência da cintura/estatura (RCE) e relação diâmetro abdominal sagital (DAS)/estatura. Homens e mulheres não diferiram em termos de circunferência na menor cintura e no ponto médio, circunferência da panturrilha (CP), dobra cutânea abdominal (DCA), relação cintura/ panturrilha e relação cintura/quadril/estatura, bem como nas medidas do DAS (Tabela 1).

Foram identificadas diferenças dentro do mesmo gênero, dependendo da faixa etária. Entre as 41 medidas antropométricas estudadas, idade foi significativamente associada com oito medidas aferidas em homens (circunferência da coxa, $\mathrm{CAB}, \mathrm{CB}, \mathrm{CMB}$, $\mathrm{DCA}, \mathrm{AMBc}$, relação cintura/coxa e índice sagital) e 28 medidas aferidas em mulheres (peso, estatura, IMC, $\mathrm{CAB}, \mathrm{CB}, \mathrm{CMB}, \mathrm{CC}$ em todos os pontos anatômicos, circunferência da coxa, do pescoço, CP, DCA, DCT, dobras cutâneas bicipital, subescapular e suprailíaca, média de seis dobras cutâneas, DAS afora no nível umbilical, AGB, AMBc, relação cintura/quadril e relação cintura/quadril/estatura).

Entre os homens, 76,4\% encontravam-se na faixa etária mais jovem (60-70 anos). Os idosos mais jovens apresentaram medidas superiores da $\mathrm{CAB}, \mathrm{CB}, \mathrm{CMB}$ e da $A M B c$, em relação aos idosos com mais de 80 anos; circunferência da coxa superior em relação aos idosos com mais de 70 anos; DCA mais espessa, e relação cintura/coxa e índice sagital menores em comparação aos homens de 71-80 anos (Tabela 2).
Entre as mulheres, $65,0 \%$ encontrava-se na faixa etária de 60-70 anos. Comparações múltiplas (teste de Bonferroni) mostraram algumas diferenças significativas entre as faixas etárias. Observou-se que, entre as idosas, as medidas de peso, $\mathrm{CAB}$ e $\mathrm{CB}$ diminuíram em relação às faixas de idade mais avançadas. As mais jovens (60-70 anos) tinham médias mais elevadas, em relação às mais velhas (>80 anos), de estatura, IMC, CC (em todos os pontos anatômicos aferidos), DCA, DCT, AGB e todas as medidas do DAS, exceto a apurada no nível umbilical, que não apresentou significância estatística. A CMB, AMBc, dobras cutâneas bicipital, subescapular, suprailíaca, média de seis dobras cutâneas, relação dobras cutâneas do tronco/dobras cutâneas das extremidades e a relação cintura/quadril foram superiores nas idosas mais jovens em relação às demais faixas etárias. Já as medidas das circunferências da coxa, do pescoço e da panturrilha de idosas de 60-80 anos foram maiores que de idosas com mais de 80 anos. Finalmente, a relação cintura/quadril/estatura foi maior nas mais idosas em relação às mais jovens (Tabela 3).

A fragilidade pôde ser avaliada em 521 idosos. A frequência de fragilidade nos idosos avaliados foi de $21,5 \%$, de pré-fragilidade $51,1 \%$, e $27,4 \%$ eram robustos. Em cada uma das categorias de fragilidade, observou-se que as medidas da estatura e altura do joelho variaram entre os estágios de fragilidade, sendo maiores entre os robustos e declinando nos estágios de pré-fragilidade e fragilidade. $\mathrm{O}$ inverso ocorreu com a medida da RCE, menor entre os robustos e aumentando entre os pré-frágeis e frágeis (Tabela 4).

Os indivíduos robustos tinham médias superiores aos frágeis nas medidas da $\mathrm{CMB}$ e da $\mathrm{AMBc}$ e superior aos pré-frágeis e frágeis na relação dobras cutâneas do tronco/dobras cutâneas das extremidades. Entretanto, os idosos robustos apresentaram médias da AGB e das dobras cutâneas da coxa e peitoral, inferiores aos préfrágeis; assim também, da DCT, das dobras cutânea bicipital, da panturrilha e da relação DAS/Estatura, em relação aos demais grupos. Por outro lado, idosos frágeis registraram as menores médias nas medidas da $\mathrm{CAB}$ e $\mathrm{CP}$ e as maiores no índice de conicidade, na relação cintura/coxa e na relação cintura/panturrilha. Tinham, também, índice sagital e CQ superiores aos robustos (Tabela 4). 
Tabela 1. Descrição das medidas antropométricas em 583 idosos do EMI-SUS, segundo o gênero.

\begin{tabular}{|c|c|c|c|c|}
\hline \multirow[b]{2}{*}{ Variáveis } & \multirow[b]{2}{*}{$\begin{array}{c}\text { Amostra total } \\
\text { (média } \pm \mathrm{DP})\end{array}$} & \multicolumn{2}{|c|}{ Gênero } & \multirow[b]{2}{*}{$\mathbf{P}$} \\
\hline & & $\begin{array}{c}\text { Masculino } \\
\text { (média } \pm D P)\end{array}$ & $\begin{array}{c}\text { Feminino } \\
\text { (média } \pm D P \text { ) }\end{array}$ & \\
\hline Peso (kg) & $70,20 \pm 14,06$ & $72,86 \pm 12,85$ & $68,67 \pm 14,51$ & 0,001 \\
\hline Estatura (m) & $1,57 \pm 0,09$ & $1,65 \pm 0,06$ & $1,52 \pm 0,07$ & $<0,001$ \\
\hline IMC (kg/m²) & $28,56 \pm 5,57$ & $26,76 \pm 4,27$ & $29,58 \pm 5,96$ & $<0,001$ \\
\hline Altura joelho (cm) & $49,35 \pm 3,22$ & $51,68 \pm 2,77$ & $48,02 \pm 2,66$ & $<0,001$ \\
\hline \multicolumn{5}{|l|}{ Circunferência (cm) } \\
\hline Antebraço & $25,97 \pm 2,65$ & $27,20 \pm 2,34$ & $25,27 \pm 2,57$ & $<0,001$ \\
\hline Braço & $31,51 \pm 4,25$ & $30,87 \pm 3,60$ & $31,88 \pm 4,54$ & 0,006 \\
\hline Muscular do braço & $25,67 \pm 3,23$ & $26,88 \pm 2,88$ & $24,97 \pm 3,23$ & $<0,001$ \\
\hline Cintura nível umbilical & $99,96 \pm 12,10$ & $97,94 \pm 9,97$ & $101,12 \pm 13,04$ & 0,003 \\
\hline Cintura menor & $93,01 \pm 11,29$ & $93,76 \pm 9,40$ & $92,58 \pm 12,23$ & 0,233 \\
\hline Cintura ponto médio* & $96,80 \pm 11,90$ & $96,79 \pm 10,20$ & $96,81 \pm 12,79$ & 0,986 \\
\hline Coxa & $48,73 \pm 6,22$ & $47,54 \pm 5,11$ & $49,41 \pm 6,69$ & 0,001 \\
\hline Panturrilha & $36,69 \pm 3,82$ & $36,61 \pm 3,64$ & $36,73 \pm 3,92$ & 0,713 \\
\hline Pescoço & $36,86 \pm 3,25$ & $39,19 \pm 2,73$ & $35,53 \pm 2,73$ & $<0,001$ \\
\hline Punho & $16,99 \pm 1,33$ & $17,67 \pm 1,12$ & $16,65 \pm 1,30$ & $<0,001$ \\
\hline Quadril & $100,61 \pm 10,89$ & $95,87 \pm 7,19$ & $103,33 \pm 11,70$ & $<0,001$ \\
\hline \multicolumn{5}{|l|}{ Dobra cutânea (mm) } \\
\hline Abdominal & $27,19 \pm 8,84$ & $26,77 \pm 8,99$ & $27,43 \pm 8,763$ & 0,399 \\
\hline Bicipital & $10,17 \pm 5,34$ & $6,56 \pm 2,97$ & $12,23 \pm 5,30$ & $<0,001$ \\
\hline Coxa & $22,07 \pm 10,92$ & $13,26 \pm 5,88$ & $27,05 \pm 9,93$ & $<0,001$ \\
\hline Panturrilha & $15,47 \pm 7,97$ & $8,52 \pm 3,79$ & $19,44 \pm 6,95$ & $<0,001$ \\
\hline Peitoral & $16,55 \pm 6,09$ & $14,95 \pm 5,25$ & $17,46 \pm 6,34$ & $<0,001$ \\
\hline Subescapular & $22,13 \pm 8,14$ & $20,08 \pm 7,02$ & $23,31 \pm 8,51$ & $<0,001$ \\
\hline Suprailíaca & $22,00 \pm 8,28$ & $19,14 \pm 7,81$ & $23,64 \pm 8,11$ & $<0,001$ \\
\hline Tricipital & $18,70 \pm 7,62$ & $12,81 \pm 5,53$ & $22,05 \pm 6,53$ & $<0,001$ \\
\hline Média de seis dobrast & $19,25 \pm 6,46$ & $15,62 \pm 5,32$ & $21,46 \pm 6,09$ & $<0,001$ \\
\hline \multicolumn{5}{|l|}{ Diâmetro Abdominal Sagital-DAS (cm) } \\
\hline Menor cintura & $24,50 \pm 3,08$ & $24,74 \pm 2,85$ & $24,36 \pm 3,21$ & 0,172 \\
\hline Maior cintura & $24,37 \pm 3,42$ & $24,30 \pm 3,24$ & $24,41 \pm 3,53$ & 0,718 \\
\hline Nível umbilical & $24,23 \pm 3,43$ & $24,12 \pm 3,23$ & $24,29 \pm 3,54$ & 0,570 \\
\hline Crista ilíaca & $24,30 \pm 3,41$ & $24,23 \pm 3,21$ & $24,34 \pm 3,53$ & 0,715 \\
\hline Ponto médio* & $24,43 \pm 3,31$ & $24,51 \pm 3,16$ & $24,38 \pm 3,39$ & 0,664 \\
\hline Posição ortostáticał & $27,80 \pm 3,96$ & $27,41 \pm 3,51$ & $28,02 \pm 4,19$ & 0,084 \\
\hline \multicolumn{5}{|l|}{ Área $\left(\mathrm{cm}^{2}\right)$} \\
\hline Gordura do braço & $27,38 \pm 13,00$ & $18,86 \pm 9,33$ & $32,23 \pm 12,28$ & $<0,001$ \\
\hline Muscular do braço corrigida & $45,48 \pm 13,09$ & $48,15 \pm 12,34$ & $43,96 \pm 13,28$ & $<0,001$ \\
\hline \multicolumn{5}{|l|}{ Relação } \\
\hline Circunferência cintura/quadril & $0,921 \pm 0,075$ & $0,974 \pm 0,054$ & $0,891 \pm 0,068$ & $<0,001$ \\
\hline Circunferência cintura/estatura & $0,618 \pm 0,082$ & $0,588 \pm 0,063$ & $0,636 \pm 0,086$ & $<0,001$ \\
\hline Circunferência cintura/coxa & $1,996 \pm 0,203$ & $2,040 \pm 00170$ & $1,971 \pm 0,215$ & $<0,001$ \\
\hline Circunferência cintura/panturrilha & $2,641 \pm 0,242$ & $2,646 \pm 0,212$ & $2,638 \pm 0,259$ & 0,706 \\
\hline Circunferência cintura/quadril/estatura & $0,013 \pm 0,003$ & $0,014 \pm 0,002$ & $0,013 \pm 0,003$ & 0,281 \\
\hline DAS/estatura & $0,155 \pm 0,023$ & $0,147 \pm 0,020$ & $0,160 \pm 0,024$ & $<0,001$ \\
\hline Dobras cutâneas do tronco/de extremidades & $1,449 \pm 0,493$ & $1,846 \pm 0,467$ & $1,208 \pm 0,325$ & $<0,001$ \\
\hline \multicolumn{5}{|l|}{ Índice } \\
\hline Sagital & $0,501 \pm 0,055$ & $0,510 \pm 0,053$ & $0,496 \pm 0,056$ & 0,004 \\
\hline Conicidade & $1,330 \pm 0,072$ & $1,339 \pm 0,062$ & $1,326 \pm 0,077$ & 0,041 \\
\hline
\end{tabular}

Teste t de Student.

* Ponto médio entre a borda superior da crista ilíaca e a borda inferior do último rebordo costal; † média das dobras cutâneas abdominal, bicipital, da panturrilha, subescapular, suprailíaca e tricipital; ₹ medido na maior proeminência do abdômen com o idoso em ortostatismo.

EMI-SUS: Estudo Epidemiológico e Clínico dos Idosos Atendidos pela Estratégia Saúde da Família do Município de Porto Alegre; DP: desvio padrão; IMC: índice de massa corporal; DAS: diâmetro abdominal sagital. 
Tabela 2. Distribuição das medidas antropométricas na amostra de 212 homens idosos, por faixa etária (EMI-SUS).

\begin{tabular}{|c|c|c|c|c|}
\hline \multirow{2}{*}{ Variáveis } & \multicolumn{3}{|c|}{ Faixa etária } & \multirow[b]{2}{*}{$\mathbf{P}$} \\
\hline & $\begin{array}{c}\text { 60-70 anos } \\
\text { (média } \pm \text { DP) }\end{array}$ & $\begin{array}{l}\text { 71-80 anos } \\
\text { (média } \pm D P \text { ) }\end{array}$ & $\begin{array}{c}>80 \text { anos } \\
\text { (média } \pm D P \text { ) }\end{array}$ & \\
\hline Frequência por faixa etária: $\mathrm{N}(\%)$ & $162(76,4)$ & $40(18,9)$ & $10(4,7)$ & \\
\hline Peso $(\mathrm{kg})$ & $73,83 \pm 12,80$ & $70,24 \pm 13,00$ & $67,00 \pm 11,00$ & 0,108 \\
\hline Estatura (m) & $1,65 \pm 0,06$ & $1,63 \pm 0,06$ & $1,65 \pm 0,06$ & 0,272 \\
\hline IMC (kg/m²) & $27,00 \pm 4,22$ & $26,28 \pm 4,56$ & $24,60 \pm 3,29$ & 0,192 \\
\hline Altura joelho (cm) & $51,63 \pm 2,81$ & $51,93 \pm 2,39$ & $51,49 \pm 3,69$ & 0,806 \\
\hline \multicolumn{5}{|l|}{ Circunferência $(\mathrm{cm})$} \\
\hline Antebraço & $27,45^{a} \pm 2,35$ & $26,56^{\mathrm{ab}} \pm 2,03$ & $25,{ }^{40 \mathrm{~b}} \pm 2,26$ & 0,008 \\
\hline Braço & $31,30^{\mathrm{a}} \pm 3,39$ & $29,85^{\mathrm{ab}} \pm 3,82$ & $27,91^{b} \pm 4,20$ & 0,002 \\
\hline Muscular do braço & $27,20^{a} \pm 2,72$ & $26,17^{\mathrm{ab}} \pm 3,11$ & $24,58^{b} \pm 3,18$ & 0,004 \\
\hline Cintura nível umbilical & $98,28 \pm 9,81$ & $97,52 \pm 10,63$ & $93,19 \pm 9,77$ & 0,357 \\
\hline Cintura menor & $94,05 \pm 9,27$ & $93,52 \pm 9,75$ & $88,64 \pm 10,18$ & 0,326 \\
\hline Cintura ponto médio* & $96,95 \pm 10,11$ & $96,63 \pm 11,15$ & $93,62 \pm 6,47$ & 0,733 \\
\hline Coxa & $48,15^{\mathrm{a}} \pm 4,98$ & $45,76^{b} \pm 4,90$ & $43,01^{b} \pm 5,35$ & 0,002 \\
\hline Panturrilha & $36,90 \pm 3,61$ & $35,82 \pm 3,58$ & $34,80 \pm 3,68$ & 0,075 \\
\hline Pescoço & $39,33 \pm 2,73$ & $38,96 \pm 2,67$ & $37,63 \pm 2,80$ & 0,190 \\
\hline Punho & $17,74 \pm 1,14$ & $17,59 \pm 1,15$ & $17,08 \pm 0,78$ & 0,419 \\
\hline Quadril & $96,21 \pm 7,12$ & $94,36 \pm 7,70$ & $96,71 \pm 5,31$ & 0,340 \\
\hline \multicolumn{5}{|l|}{ Dobra cutânea (mm) } \\
\hline Abdominal & $27,94^{\mathrm{a}} \pm 9,20$ & $23,01^{b} \pm 7,31$ & $22,50^{\mathrm{ab}} \pm 6,05$ & 0,003 \\
\hline Bicipital & $6,66 \pm 3,07$ & $6,49 \pm 2,68$ & $4,81 \pm 1,71$ & 0,228 \\
\hline Coxa & $13,57 \pm 6,16$ & $12,00 \pm 4,51$ & $13,00 \pm 5,74$ & 0,343 \\
\hline Panturrilha & $8,45 \pm 3,65$ & $8,68 \pm 4,30$ & $9,19 \pm 4,26$ & 0,833 \\
\hline Peitoral & $15,32 \pm 5,11$ & $13,86 \pm 5,77$ & $12,94 \pm 4,51$ & 0,159 \\
\hline Subescapular & $20,53 \pm 6,89$ & $19,18 \pm 7,42$ & $16,35 \pm 6,72$ & 0,126 \\
\hline Suprailíaca & $19,55 \pm 7,72$ & $17,74 \pm 8,37$ & $18,00 \pm 6,65$ & 0,399 \\
\hline Tricipital & $13,13 \pm 5,58$ & $11,71 \pm 5,28$ & $11,78 \pm 5,55$ & 0,297 \\
\hline Média de seis dobrast & $16,09 \pm 5,40$ & $14,51 \pm 4,99$ & $12,52 \pm 4,09$ & 0,174 \\
\hline \multicolumn{5}{|l|}{ Diâmetro Abdominal Sagital-DAS (cm) } \\
\hline Menor cintura & $24,79 \pm 2,82$ & $24,64 \pm 3,12$ & $23,78 \pm 1,83$ & 0,683 \\
\hline Maior cintura & $24,36 \pm 3,22$ & $24,12 \pm 3,58$ & $23,63 \pm 1,79$ & 0,817 \\
\hline Nível umbilical & $24,13 \pm 3,22$ & $24,14 \pm 3,48$ & $23,53 \pm 1,86$ & 0,905 \\
\hline Crista ilíaca & $24,24 \pm 3,22$ & $24,33 \pm 3,36$ & $23,32 \pm 1,94$ & 0,772 \\
\hline Ponto médio* & $24,56 \pm 3,17$ & $24,42 \pm 3,34$ & $23,65 \pm 1,73$ & 0,776 \\
\hline Posição ortostáticał & $27,41 \pm 3,45$ & $27,50 \pm 3,94$ & $26,72 \pm 2,69$ & 0,880 \\
\hline \multicolumn{5}{|l|}{ Área $\left(\mathrm{cm}^{2}\right)$} \\
\hline Gordura do braço & $19,53 \pm 9,52$ & $16,79 \pm 8,39$ & $16,05 \pm 8,82$ & 0,163 \\
\hline Muscular do braço corrigida & $49,44^{\mathrm{a}} \pm 11,83$ & $45,26^{\mathrm{ab}} \pm 13,24$ & $38,80^{b} \pm 12,02$ & 0,007 \\
\hline \multicolumn{5}{|l|}{ Relação } \\
\hline Circunferência cintura/quadril & $0,97 \pm 0,06$ & $0,98 \pm 0,05$ & $0,95 \pm 0,05$ & 0,256 \\
\hline Circunferência cintura/estatura & $0,59 \pm 0,06$ & $0,59 \pm 0,07$ & $0,57 \pm 0,03$ & 0,608 \\
\hline Circunferência cintura/coxa & $2,02^{\mathrm{b}} \pm 0,17$ & $2,12^{\mathrm{a}} \pm 0,16$ & $2,08^{\mathrm{ab}} \pm 0,10$ & 0,006 \\
\hline Circunferência cintura/panturrilha & $2,63 \pm 0,21$ & $2,70 \pm 0,22$ & $2,62 \pm 0,18$ & 0,166 \\
\hline Circunferência cintura/quadril/estatura & $0,013 \pm 0,002$ & $0,014 \pm 0,002$ & $0,014 \pm 0,0015$ & 0,054 \\
\hline DAS/estatura & $0,147 \pm 0,020$ & $0,149 \pm 0,022$ & $0,142 \pm 0,012$ & 0,691 \\
\hline Dobras cutâneas do tronco/de extremidades & $1,872 \pm 0,459$ & $1,803 \pm 0,504$ & $1,592 \pm 0,399$ & 0,375 \\
\hline \multicolumn{5}{|l|}{ Índice } \\
\hline Sagital & $0,505^{\mathrm{b}} \pm 0,052$ & $0,534^{a} \pm 0,054$ & $0,518^{\mathrm{ab}} \pm 0,026$ & 0,014 \\
\hline Conicidade & $1,335 \pm 0,064$ & $1,356 \pm 0,056$ & $1,327 \pm 0,031$ & 0,130 \\
\hline
\end{tabular}

Análise de variância ANOVA. Valores médios com letras diferentes subscritas são significativamente diferentes (teste de Bonferroni).

* Ponto médio entre a borda superior da crista ilíaca e a borda inferior do último rebordo costal; † média das dobras cutâneas abdominal, bicipital, da panturrilha, subescapular, suprailíaca e tricipital; ‡ medido na maior proeminência do abdômen com o idoso em ortostatismo.

EMI-SUS: Estudo Epidemiológico e Clínico dos Idosos Atendidos pela Estratégia Saúde da Família do Município de Porto Alegre; IMC: índice de massa corporal; DAS: diâmetro abdominal sagital. 
Tabela 3. Distribuição das medidas antropométricas na amostra de 371 mulheres idosas, por faixa etária (EMI-SUS).

\begin{tabular}{|c|c|c|c|c|}
\hline \multirow[b]{2}{*}{ Variáveis } & \multicolumn{3}{|c|}{ Faixa etária } & \multirow[b]{2}{*}{$\mathbf{P}$} \\
\hline & $\begin{array}{l}\text { 60-70 anos } \\
\text { (média } \pm \text { DP) }\end{array}$ & $\begin{array}{l}\text { 71-80 anos } \\
\text { (média } \pm \text { DP) }\end{array}$ & $\begin{array}{c}>80 \text { anos } \\
\text { (média } \pm \text { DP) }\end{array}$ & \\
\hline Frequência por faixa etária: $\mathrm{N}(\%)$ & $241(65,0)$ & $102(27,5)$ & $28(7,5)$ & \\
\hline Peso $(k g)$ & $70,71^{\mathrm{a}} \pm 13,81$ & $66,62^{\mathrm{b}} \pm 14,93$ & $57,91^{c} \pm 13,72$ & $<0,001$ \\
\hline Estatura $(m)$ & $1,53^{a} \pm 0,06$ & $1,52^{\mathrm{ab}} \pm 0,07$ & $1,48^{\mathrm{b}} \pm 0,07$ & 0,001 \\
\hline $\mathrm{IMC}\left(\mathrm{kg} / \mathrm{m}^{2}\right)$ & $30,19^{a} \pm 5,81$ & $28,97^{b} \pm 5,95$ & $26,41^{b} \pm 6,28$ & 0,004 \\
\hline Altura joelho $(\mathrm{cm})$ & $48,05 \pm 2,69$ & $48,06 \pm 2,65$ & $47,62 \pm 2,48$ & 0,718 \\
\hline \multicolumn{5}{|l|}{ Circunferência (cm) } \\
\hline Antebraço & $25,66^{\mathrm{a}} \pm 2,19$ & $24,93^{b} \pm 2,96$ & $22,97 c \pm 2,86$ & $<0,001$ \\
\hline Braço & $32,62^{\mathrm{a}} \pm 4,12$ & $31,05^{b} \pm 4,78$ & $28,63^{c} \pm 5,31$ & $<0,001$ \\
\hline Muscular do braço & $25,50^{a} \pm 3,03$ & $24,32^{\mathrm{b}} \pm 3,38$ & $22,83^{b} \pm 3,06$ & $<0,001$ \\
\hline Cintura nível umbilical & $102,16^{\mathrm{a}} \pm 12,60$ & $99,77^{\mathrm{ab}} \pm 13,64$ & $95,68^{\mathrm{b}} \pm 13,83$ & 0,045 \\
\hline Cintura menor & $93,94^{a} \pm 11,98$ & $90,67^{\mathrm{ab}} \pm 12,32$ & $85,92^{\mathrm{b}} \pm 11,94$ & 0,003 \\
\hline Cintura ponto médio* & $98,24^{\mathrm{a}} \pm 12,48$ & $94,76^{\mathrm{ab}} \pm 13,01$ & $89,89^{b} \pm 12,30$ & 0,003 \\
\hline Coxa & $50,03^{a} \pm 6,35$ & $48,86^{a} \pm 6,75$ & $45,07^{b} \pm 8,36$ & 0,002 \\
\hline Panturrilha & $37,30^{a} \pm 3,60$ & $36,23^{a} \pm 4,17$ & $33,28^{b} \pm 3,95$ & $<0,001$ \\
\hline Pescoço & $35,79^{a} \pm 2,63$ & $35,32^{\mathrm{a}} \pm 2,96$ & $33,81^{b} \pm 2,05$ & 0,002 \\
\hline Punho & $16,68 \pm 1,19$ & $16,82 \pm 1,50$ & $16,01 \pm 1,40$ & 0,070 \\
\hline Quadril & $104,00 \pm 11,61$ & $102,57 \pm 11,21$ & $98,89 \pm 14,27$ & 0,131 \\
\hline \multicolumn{5}{|l|}{ Dobra cutânea (mm) } \\
\hline Abdominal & $28,49^{a} \pm 8,42$ & $26,01^{\mathrm{ab}} \pm 8,86$ & $21,95^{b} \pm 9,55$ & 0,001 \\
\hline Bicipital & $13,02^{\mathrm{a}} \pm 5,25$ & $11,00^{b} \pm 5,09$ & $9,77^{b} \pm 5,01$ & $<0,001$ \\
\hline Coxa & $27,28 \pm 9,80$ & $27,00 \pm 10,01$ & $24,68 \pm 11,08$ & 0,501 \\
\hline Panturrilha & $19,27 \pm 6,69$ & $20,08 \pm 7,23$ & $18,44 \pm 8,30$ & 0,478 \\
\hline Peitoral & $17,91 \pm 6,40$ & $16,97 \pm 5,87$ & $15,16 \pm 7,25$ & 0,079 \\
\hline Subescapular & $25,00^{a} \pm 8,26$ & $20,97^{b} \pm 7,68$ & $17,34^{b} \pm 9,07$ & $<0,001$ \\
\hline Suprailíaca & $25,08^{a} \pm 7,79$ & $21,29^{b} \pm 7,96$ & $18,12^{\mathrm{b}} \pm 7,75$ & $<0,001$ \\
\hline Tricipital & $22,64^{a} \pm 6,14$ & $21,44^{\mathrm{ab}} \pm 6,79$ & $19,15^{b} \pm 8,03$ & 0,016 \\
\hline Média de seis dobrast & $22,44^{\mathrm{a}} \pm 6,11$ & $19,86^{b} \pm 5,49$ & $16,68^{b} \pm 4,83$ & 0,001 \\
\hline \multicolumn{5}{|l|}{ Diâmetro Abdominal Sagital-DAS (cm) } \\
\hline Menor cintura & $24,62^{\mathrm{a}} \pm 3,02$ & $24,07^{\mathrm{ab}} \pm 3,59$ & $22,63^{b} \pm 3,00$ & 0,020 \\
\hline Maior cintura & $24,71^{\mathrm{a}} \pm 3,33$ & $24,02^{\mathrm{ab}} \pm 3,91$ & $22,67^{b} \pm 3,41$ & 0,025 \\
\hline Nível umbilical & $24,54 \pm 3,38$ & $24,01 \pm 3,87$ & $22,69 \pm 3,40$ & 0,062 \\
\hline Crista ilíaca & $24,65^{a} \pm 3,38$ & $23,98^{\mathrm{ab}} \pm 3,82$ & $22,43^{b} \pm 3,19$ & 0,015 \\
\hline Ponto médio* & $24,66^{\mathrm{a}} \pm 3,21$ & $24,06^{\mathrm{ab}} \pm 3,77$ & $22,56^{b} \pm 3,00$ & 0,019 \\
\hline Posição ortostáticał & $28,31^{\mathrm{a}} \pm 3,98$ & $27,74^{\mathrm{ab}} \pm 4,53$ & $25,93^{b} \pm 4,47$ & 0,043 \\
\hline \multicolumn{5}{|l|}{ Área $\left(\mathrm{cm}^{2}\right)$} \\
\hline Gordura do braço & $33,51^{\mathrm{a}} \pm 11,85$ & $30,83^{\mathrm{ab}} \pm 12,23$ & $26,13^{\mathrm{b}} \pm 14,17$ & 0,005 \\
\hline Muscular do braço corrigida & $45,98^{\mathrm{a}} \pm 13,02$ & $41,45^{b} \pm 13,17$ & $35,69^{b} \pm 11,49$ & $<0,001$ \\
\hline \multicolumn{5}{|l|}{ Relação } \\
\hline Circunferência cintura/quadril & $0,90^{\mathrm{a}} \pm 0,07$ & $0,88^{b} \pm 0,07$ & $0,86^{b} \pm 0,06$ & 0,005 \\
\hline Circunferência cintura/estatura & $0,64 \pm 0,08$ & $0,63 \pm 0,09$ & $0,61 \pm 0,09$ & 0,091 \\
\hline Circunferência cintura/coxa & $1,97 \pm 0,21$ & $1,96 \pm 0,23$ & $1,99 \pm 0,19$ & 0,709 \\
\hline Circunferência cintura/panturrilha & $2,64 \pm 0,25$ & $2,62 \pm 0,27$ & $2,71 \pm 0,27$ & 0,424 \\
\hline Circunferência cintura/quadril/estatura & $0,013^{\mathrm{b}} \pm 0,002$ & $0,014^{\mathrm{ab}} \pm 0,003$ & $0,015^{\mathrm{a}} \pm 0,005$ & 0,040 \\
\hline DAS/estatura & $0,161 \pm 0,023$ & $0,158 \pm 0,026$ & $0,152 \pm 0,023$ & 0,176 \\
\hline Dobras cutâneas do tronco/de extremidades & $1,276^{\mathrm{a}} \pm 0,330$ & $1,066^{\mathrm{b}} \pm 0,255$ & $1,026^{b} \pm 0,281$ & $<0,001$ \\
\hline \multicolumn{5}{|l|}{ Índice } \\
\hline Sagital & $0,497 \pm 0,053$ & $0,494 \pm 0,064$ & $0,499 \pm 0,043$ & 0,892 \\
\hline Conicidade & $1,330 \pm 0,075$ & $1,318 \pm 0,082$ & $1,314 \pm 0,067$ & 0,348 \\
\hline
\end{tabular}

Análise de variância ANOVA. Valores médios com letras diferentes subscritas são significativamente diferentes (teste de Bonferroni).

* Ponto médio entre a borda superior da crista ilíaca e a borda inferior do último rebordo costal; † média das dobras cutâneas abdominal, bicipital, da panturrilha, subescapular, suprailíaca e tricipital; ¥ medido na maior proeminência do abdômen com o idoso em ortostatismo.

EMI-SUS: Estudo Epidemiológico e Clínico dos Idosos Atendidos pela Estratégia Saúde da Família do Município de Porto Alegre; IMC: índice de massa corporal; DAS: diâmetro abdominal sagital. 
Tabela 4. Associação entre medidas antropométricas e fragilidade em uma amostra de 521 idosos do EMI-SUS.

\begin{tabular}{|c|c|c|c|c|}
\hline \multirow{2}{*}{ Variáveis } & \multicolumn{3}{|c|}{ Fragilidade } & \multirow[b]{2}{*}{$\mathbf{P}$} \\
\hline & $\begin{array}{c}\text { Robusto } \\
\text { (média } \pm \text { DP) }\end{array}$ & $\begin{array}{c}\text { Pré-Frágil } \\
\text { (média } \pm D P)\end{array}$ & $\begin{array}{c}\text { Frágil } \\
\text { (média } \pm D P)\end{array}$ & \\
\hline Frequência de fragilidade: $\mathrm{N}(\%)$ & $143(27,4)$ & $266(51,1)$ & $112(21,5)$ & \\
\hline Peso $(k g)$ & $71,40 \pm 11,59$ & $70,55 \pm 15,12$ & $67,81 \pm 14,37$ & 0,114 \\
\hline Estatura (m) & $1,60^{\mathrm{a}} \pm 0,08$ & $1,57^{b} \pm 0,09$ & $1,53^{c} \pm 0,09$ & $<0,001$ \\
\hline IMC $\left(\mathrm{kg} / \mathrm{m}^{2}\right)$ & $27,83 \pm 4,29$ & $28,75 \pm 5,82$ & $29,15 \pm 6,41$ & 0,138 \\
\hline Altura joelho (cm) & $50,24^{a} \pm 3,05$ & $49,29^{b} \pm 3,18$ & $48,25^{\complement} \pm 3,10$ & $<0,001$ \\
\hline \multicolumn{5}{|l|}{ Circunferência (cm) } \\
\hline Antebraço & $26,51^{\mathrm{a}} \pm 2,49$ & $26,05^{a} \pm 2,71$ & $24,97^{b} \pm 2,40$ & $<0,001$ \\
\hline Braço & $31,62 \pm 3,19$ & $31,70 \pm 4,39$ & $30,70 \pm 4,75$ & 0,094 \\
\hline Muscular do braço & $26,35^{\mathrm{a}} \pm 2,92$ & $25,58^{\mathrm{ab}} \pm 3,25$ & $24,73^{b} \pm 3,18$ & $<0,001$ \\
\hline Cintura nível umbilical & $98,64 \pm 9,31$ & $100,09 \pm 12,89$ & $102,20 \pm 12,96$ & 0,083 \\
\hline Cintura menor & $92,46 \pm 9,27$ & $93,10 \pm 12,26$ & $94,12 \pm 11,41$ & 0,549 \\
\hline Cintura ponto médio* & $95,88 \pm 9,31$ & $96,95 \pm 12,99$ & $98,26 \pm 12,35$ & 0,326 \\
\hline Coxa & $48,82 \pm 5,40$ & $49,06 \pm 6,52$ & $48,03 \pm 6,26$ & 0,391 \\
\hline Panturrilha & $37,11^{\mathrm{a}} \pm 3,41$ & $36,89^{a} \pm 3,96$ & $35,76^{b} \pm 3,96$ & 0,012 \\
\hline Pescoço & $37,28 \pm 3,19$ & $36,77 \pm 3,36$ & $36,56 \pm 3,07$ & 0,178 \\
\hline Punho & $17,06 \pm 1,21$ & $17,12 \pm 1,45$ & $16,70 \pm 1,27$ & 0,155 \\
\hline Quadril & $98,91^{b} \pm 8,24$ & $101,14^{\mathrm{ab}} \pm 11,39$ & $102,18^{\mathrm{a}} \pm 12,44$ & 0,049 \\
\hline \multicolumn{5}{|l|}{ Dobra cutânea (mm) } \\
\hline Abdominal & $27,22 \pm 8,20$ & $27,61 \pm 9,08$ & $26,85 \pm 8,75$ & 0,751 \\
\hline Bicipital & $8,92^{\mathrm{b}} \pm 4,37$ & $10,59^{a} \pm 5,60$ & $10,64^{a} \pm 5,54$ & 0,005 \\
\hline Coxa & $20,05^{b} \pm 10,18$ & $23,11^{\mathrm{a}} \pm 11,50$ & $22,70^{\mathrm{ab}} \pm 10,21$ & 0,023 \\
\hline Panturrilha & $13,27^{\mathrm{b}} \pm 7,21$ & $16,15^{\mathrm{a}} \pm 8,10$ & $16,78^{a} \pm 8,18$ & $<0,001$ \\
\hline Peitoral & $15,55^{b} \pm 5,45$ & $17,23^{a} \pm 6,31$ & $16,59^{\mathrm{ab}} \pm 6,03$ & 0,027 \\
\hline Subescapular & $21,92 \pm 7,00$ & $22,71 \pm 8,11$ & $20,64 \pm 8,53$ & 0,066 \\
\hline Suprailíaca & $21,48 \pm 7,54$ & $22,63 \pm 8,55$ & $21,42 \pm 8,62$ & 0,287 \\
\hline Tricipital & $16,77^{b} \pm 6,74$ & $19,49^{\mathrm{a}} \pm 7,63$ & $19,19^{a} \pm 7,93$ & 0,002 \\
\hline Média de 6 dobrast & $18,42 \pm 5,20$ & $19,77 \pm 6,72$ & $18,65 \pm 6,66$ & 0,252 \\
\hline \multicolumn{5}{|l|}{ Diâmetro Abdominal Sagital-DAS (cm) } \\
\hline Menor cintura & $24,35 \pm 2,56$ & $24,50 \pm 3,26$ & $24,79 \pm 3,40$ & 0,606 \\
\hline Maior cintura & $24,05 \pm 2,83$ & $24,47 \pm 3,57$ & $24,75 \pm 3,87$ & 0,302 \\
\hline Nível umbilical & $23,85 \pm 2,87$ & $24,33 \pm 3,53$ & $24,72 \pm 3,90$ & 0,171 \\
\hline Crista ilíaca & $23,95 \pm 2,78$ & $24,42 \pm 3,56$ & $24,68 \pm 3,87$ & 0,256 \\
\hline Ponto médio* & $24,13 \pm 2,74$ & $24,51 \pm 3,48$ & $24,80 \pm 3,60$ & 0,321 \\
\hline Posição ortostáticał & $27,29 \pm 3,29$ & $27,93 \pm 4,14$ & $28,49 \pm 4,43$ & 0,081 \\
\hline \multicolumn{5}{|l|}{ Área $\left(\mathrm{cm}^{2}\right)$} \\
\hline Gordura do braço & $24,44^{\mathrm{b}} \pm 10,54$ & $28,69^{a} \pm 13,30$ & $27,58^{\mathrm{ab}} \pm 13,77$ & 0,006 \\
\hline Muscular do braço corrigida & $47,58^{\mathrm{a}} \pm 11,54$ & $45,30^{\mathrm{ab}} \pm 13,13$ & $42,07^{b} \pm 13,10$ & 0,003 \\
\hline \multicolumn{5}{|l|}{ Relação circunferência } \\
\hline Cintura/quadril & $0,931 \pm 0,070$ & $0,917 \pm 0,078$ & $0,921 \pm 0,075$ & 0,216 \\
\hline Cintura/estatura & $0,599^{c} \pm 0,063$ & $0,620^{b} \pm 0,085$ & $0,646^{a} \pm 0,090$ & $<0,001$ \\
\hline Cintura/coxa & $1,973^{b} \pm 0,171$ & $1,983^{b} \pm 0,209$ & $2,066^{a} \pm 0,231$ & 0,001 \\
\hline Cintura/panturrilha & $2,588^{\mathrm{b}} \pm 0,207$ & $2,628^{\mathrm{b}} \pm 0,244$ & $2,751^{\mathrm{a}} \pm 0,270$ & $<0,001$ \\
\hline Cintura/quadril/estatura & $0,013 \pm 0,002$ & $0,014 \pm 0,003$ & $0,014 \pm 0,003$ & 0,647 \\
\hline Relação DAS/estatura & $0,150^{b} \pm 0,019$ & $0,156^{\mathrm{a}} \pm 0,023$ & $0,162^{a} \pm 0,028$ & $<0,001$ \\
\hline Relação dobras cutâneas do tronco/ de extremidades & $1,606^{a} \pm 0,543$ & $1,413^{\mathrm{b}} \pm 0,482$ & $1,331^{\mathrm{b}} \pm 0,451$ & 0,004 \\
\hline \multicolumn{5}{|l|}{ Índice } \\
\hline Sagital & $0,493^{b} \pm 0,050$ & $0,501^{\mathrm{ab}} \pm 0,064$ & $0,516^{a} \pm 0,064$ & 0,015 \\
\hline Conicidade & $1,320^{b} \pm 0,063$ & $1,328^{\mathrm{b}} \pm 0,077$ & $1,355^{a} \pm 0,072$ & 0,001 \\
\hline
\end{tabular}

Análise de variância ANOVA. Valores médios com letras diferentes subscritas são significativamente diferentes (teste de Bonferroni).

* Ponto médio entre a borda superior da crista ilíaca e a borda inferior do último rebordo costal; † média das dobras cutâneas abdominal, bicipital, da panturrilha, subescapular, suprailíaca e tricipital; キ medido na maior proeminência do abdômen com o idoso em ortostatismo.

EMI-SUS: Estudo Epidemiológico e Clínico dos Idosos Atendidos pela Estratégia Saúde da Família do Município de Porto Alegre; IMC: índice de massa corporal; DAS: diâmetro abdominal sagital. 


\section{DISCUSSÃO}

Neste estudo, foram descritas 41 medidas antropométricas de idosos que vivem na comunidade e que são atendidos pelo sistema de saúde pública, sendo analisada sua associação com gênero, idade e fragilidade. $\mathrm{Na}$ avaliação das medidas antropométricas segundo o gênero, as idosas apresentaram médias mais elevadas em relação aos homens, em medidas de obesidade geral (IMC), de obesidade central (CC e CQ, RCE e relação DAS/Estatura) e em medidas de reserva adiposa (dobras cutâneas, média de seis dobras cutâneas, $\mathrm{CB}$ e AGB) associadas a risco cardiovascular e desordens metabólicas [42].

Lipschitz [43], em 1994, propôs uma classificação para o estado nutricional que considera as modificações na composição corporal dos idosos, recomendando os pontos de corte abaixo de $22 \mathrm{~kg} / \mathrm{m}^{2}$ para baixo peso e acima de $27 \mathrm{~kg} / \mathrm{m}^{2}$ para sobrepeso, referência que indica sobrepeso em nossa amostra total $\left(28,56 \pm 5,57 \mathrm{~kg} / \mathrm{m}^{2}\right)$. O maior IMC das mulheres em relação ao dos homens $\left(29,58 \pm 5,96\right.$ vs. $\left.26,76 \pm 4,27 \mathrm{~kg} / \mathrm{m}^{2}\right)$ já vem sendo demonstrado em estudos anteriores [1,2,44-45,46]. Mulheres, em geral, apresentam maior prevalência de obesidade [44] e parecem ter, diferentemente dos homens, o IMC como indicativo de adiposidade [47].

Medidas de dobras cutâneas, por sua vez, são frequentemente maiores nas mulheres. Outros autores já confirmaram estes resultados em relação às DCT [1,2], bicipital $[48,49]$ subescapular $[1,47,48]$, suprailíaca [1,48], da panturrilha [48] e da coxa [1]. Medidas de dobras cutâneas possibilitam estimar gordura subcutânea com bastante acurácia, e as medidas da DCT e subescapular vêm sendo utilizadas como indicadores de reserva adiposa [50]. As demais dobras, entretanto, são usadas em equações preditivas e não existem parâmetros para sua avaliação isoladamente $[51,52]$. Os resultados deste estudo fornecem dados que poderão ser comparados em estudos futuros.

Valores médios superiores da CB $[2,46]$ e da AGB [47], e menores da CMB [2] parecem sugerir maior adiposidade subcutânea e menor reserva de massa muscular entre as mulheres. Com o envelhecimento, ocorrem alterações na composição corporal, com o aumento do tecido adiposo e diminuição da massa livre de gordura [53]. Nesta amostra, esta tendência parece ser mais evidente entre as mulheres, colocando-as em maior risco de obesidade sarcopênica [9].

A medida da $\mathrm{CC}$, independentemente do ponto anatômico em que é aferido, normalmente é maior entre os homens $[1,44,48]$ entretanto, quando aferida no nível umbilical, esta medida foi superior entre as mulheres. Nos últimos anos, tem havido muitos questionamentos quanto à escolha do índice antropométrico de adiposidade mais preciso (para fins de pesquisa) e mais conveniente (para a prática clínica). Estudo recente sugere o uso combinado de IMC e CC, pois indica que a CC permite estratificar os indivíduos considerando o nível de adiposidade visceral e o perfil cardiovascular, para um dado IMC [54]. Mulheres, no presente estudo, mostraram níveis elevados de IMC $\left(29,58 \pm 5,96 \mathrm{~kg} / \mathrm{m}^{2}\right)$ e de CC $(101,12 \pm 13,04 \mathrm{~cm})$, cenário representativo de risco associado a complicações cardiovasculares e metabólicas. A gordura visceral é apenas mais um dos muitos locais de depósito de gordura ectópica usados pelo organismo, quando o tecido adiposo subcutâneo não consegue acomodar o excesso de gordura por sua limitada expansão [55]. Mulheres, ainda, mais frequentemente que os homens, apresentam uma reserva de gordura subcutânea nos glúteos, e a CQ aumentada em relação aos homens foi observada neste estudo e em pesquisas prévias $[1,44,48,56]$.

Medidas de CC aumentada e CQ diminuída, em conjunto, estão associadas com aumento do risco de doenças cardiovasculares. Os reais efeitos da obesidade visceral podem ser subestimados quando a CQ não é considerada [57]. Embora sejam medidas correlacionadas, os efeitos da adiposidade gluteofemoral subcutânea na saúde metabólica são diferentes dos da adiposidade visceral $[13,58]$, havendo possibilidade de CQ aumentada refletir maior massa muscular. Uma revisão mostra, inclusive, benefícios protetores relacionados ao perfil glicêmico e lipídico, e à diminuição do risco cardiovascular e metabólico[58]. Assim como a CQ, a medida da circunferência da coxa, maior entre as mulheres, também tem sido associada com menor risco de diabetes mellitus tipo 2 [59].

Por outro lado, homens tinham médias superiores às das mulheres em medidas que avaliam tamanho, proporção e compleição (peso, estatura, altura do joelho, CAB, circunferência do punho), como era esperado, pois estudos brasileiros [2,47] americanos $[45,49,53,56,60]$ e europeus [1] já trazem informações da superioridade de peso e estatura dos homens em relação às mulheres. Assim, também, medidas maiores da altura do joelho $[56,48]$.

Estudos avaliando medidas de circunferência do punho e $\mathrm{CAB}$ em relação ao gênero não foram localizados, talvez por serem perímetros com evidências biológicas ainda não tão bem estabelecidas [42]. Entretanto, já há registro na literatura de que alterações na $\mathrm{CAB}$ podem estar associadas à força de preensão palmar, principalmente entre os homens [61], o que 
poderia explicar o fato de homens apresentarem $\mathrm{CAB}$ maior que as mulheres.

Homens também tiveram médias mais elevadas de medidas relacionadas a situações de risco cardiovascular, metabólico e de apneia obstrutiva do sono $[62,63]$ como a circunferência do pescoço [64], a relação cintura/quadril $[1,47,56,64]$, os índices sagital [36], e de conicidade [65]. Homens apresentam apneia obstrutiva do sono mais severa do que as mulheres e a medida da circunferência do pescoço aumentada é um importante preditor desta severidade [64]. O risco de sofrer apneia obstrutiva severa é maior em homens com relação cintura/quadril >1 [66].

A relação cintura/coxa e o índice sagital foram propostos como medidas alternativas à relação cintura/ quadril, para verificação da distribuição de gordura corporal e predição de morbidades. Isto porque ambos os índices utilizam o perímetro da coxa, medida que não seria afetada por variações na arquitetura pélvica, como a CQ [35]. Em homens americanos de meia idade, o índice sagital foi considerado a mais poderosa medida de risco de doenças cardiovasculares [67]. Não foram encontrados estudos que avaliaram a relação cintura/coxa e a relação das dobras cutâneas do tronco/dobras cutâneas das extremidades em idosos da comunidade. São medidas ainda pouco estudadas e o interesse pela relação entre a adiposidade dos membros e a adiposidade do tronco foi a partir de um estudo que demonstrou forte correlação entre esta razão (com medidas avaliadas através do DEXA) e a resistência à insulina em idosos [37].

Também já há registros na literatura sobre a superioridade dos homens em relação às medidas indicativas de reserva de tecido muscular [68], como a $\mathrm{CMB}$ [2], e a $\mathrm{AMBc}[47,48]$. Massa muscular tem estreita relação com força muscular. Homens apresentam mais massa magra que mulheres, seja total ou apendicular, e essa diferença é acentuada nas idades mais avançadas. Maior reserva de massa muscular nos homens pode se constituir em fator protetor para as complicações associadas ao ciclo da fragilidade [68].

A RCE é indicativa de risco de obesidade, tanto em homens como em mulheres, em diferentes grupos étnicos [69]. Entre os homens desta amostra este índice foi menor, assim como em dois estudos realizados no Brasil [46,70]. Entretanto, homens e mulheres apresentaram RCE $>0,5$, ponto de corte indicador de risco para ambos os gêneros $[65,70]$.

Nenhuma das medidas do DAS diferenciou-se segundo o gênero, resultado similar ao encontrado no estudo Hoorn [71]. Entretanto, em amostras de adultos brasileiros, os resultados foram contro- versos [72,73]. O DAS tem sido associado ao risco de doenças crônicas, mas essas relações dependem da população estudada e dos variados pontos anatômicos utilizados nos métodos [74,75] Estes aspectos podem justificar as diferenças entre os dados do presente estudo e de estudos prévios.

A CP $>31 \mathrm{~cm}$ tem sido associada com maior força muscular, melhor desempenho físico e funcional [12]. No presente estudo a CP média foi $36,7 \pm 3,8 \mathrm{~cm}$ e não se mostrou diferente entre os gêneros. Encontramos três estudos que concordam com este resultado $[46,56,76]$ e outro discordante, no qual a CP foi maior entre os homens, sendo que a média de idade dos homens desse estudo discordante era inferior aos demais, o que talvez explique a diferença encontrada [48].

Quando consideradas as alterações nas medidas antropométricas em relação ao avanço da idade, os resultados encontrados sugerem que mudanças corporais continuam ocorrendo durante todo o processo do envelhecimento. Entretanto, essas modulações nas dimensões corporais parecem ser diferenciadas na sua magnitude $[45,60]$, no tempo em que ocorrem e de acordo com o gênero [45]. As alterações nas medidas antropométricas, tanto dos homens como das mulheres, ocorreram, mais frequentemente, entre os mais jovens (60-70 anos) em relação aos mais velhos (80 anos ou mais), acompanhando dados da literatura $[2,60]$.

Quanto ao gênero, como já observado em estudos anteriores, as modificações ocorridas foram mais frequentes entre as mulheres [47] e podem estar associadas à perda de massa muscular e à redução e redistribuição do tecido adiposo, mais marcantes em mulheres, e mais percebidas a partir dos 70 anos $[45,53]$.

$\mathrm{Na}$ avaliação das medidas de tamanho, proporção e compleição, verificou-se que o peso das mulheres diminuiu em cada faixa etária, como já observado na literatura $[45,53,60,71,77]$, mas não foi observada diferença significativa de peso entre os homens $[47,77]$. Em geral, o peso aumenta na idade adulta e decresce no envelhecimento (mais ou menos $1 \mathrm{~kg}$ por década). As alterações no peso, entretanto, não se comportam da mesma forma entre os sexos, e alguns estudos relatam uma diminuição menor de peso entre os homens $[1,2,53]$. Outros, diferentemente, verificaram perda de peso tanto em homens e mulheres, com o decorrer do tempo $[45,47]$.

A redução na estatura tem sido relacionada com alterações no músculo esquelético, como diminuição da água corporal [11], redução da massa muscular [8], desmineralização e diminuição dos espaços intervertebrais [56]. Nas mulheres, essa diminuição parece 
ser mais rápida do que entre os homens, o que pode ser explicado pela maior prevalência de osteoporose entre elas [78,79]. Para indivíduos com idade igual ou superior a 60 anos, nos quais, por alguma(s) dessa(s) alterações, a altura já tenha sofrido diminuição, a estatura estimada pode ser uma alternativa confiável $[21,80]$. Por outro lado, sabe-se que o tamanho dos ossos longos do corpo não se altera com a idade [50]. Assim, a altura do joelho, diferentemente da estatura, tem se mostrado uma medida com menores alterações em relação ao envelhecimento [1,60], e isso foi confirmado neste estudo e em outras pesquisas previamente realizadas $[60,77]$.

A CAB foi menor em todas as faixas etárias nas mulheres e nos homens mais velhos em relação aos mais jovens. A literatura descreve uma diminuição da $\mathrm{CAB}$ dominante, com a idade, porém menos marcante nas mulheres [61], entretanto sem evidências biológicas estabelecidas [42].

Entre as medidas relacionadas à obesidade geral e central, observou-se que mulheres mais velhas apresentaram IMC inferior às mais novas, porém entre os homens o índice não decresceu, e este achado foi raro na literatura [47,77]. A maioria dos estudos observou diminuição do IMC em ambos os gêneros $[1,2,45,56]$ e um estudo registrou IMC menor em idosos com 80 anos ou mais [46]. Estas discordâncias já foram salientadas por outros autores e têm sido atribuídas a diferenças étnicas, socioeconômicas e de estilo de vida, entre outras $[2,46]$.

A CC mostrou-se diminuída nas mulheres mais velhas em relação às mais novas, em três pontos anatômicos, enquanto que nos homens não houve diferença. Houve controvérsias entre sete estudos que realizaram esta avaliação. Um deles [47] corrobora com os presentes achados, dois observaram redução em ambos os gêneros [45,60], outros dois somente diminuição nos homens $[1,2]$ e os dois últimos nenhuma redução na CC com o passar dos anos [46,77]. As mudanças relacionadas ao acúmulo de gordura visceral associado ao envelhecimento podem ser afetadas tanto pela quantidade de tecido adiposo préexistente no indivíduo adulto, como pelo aumento da massa corporal [47]. Gênero, características genéticas e fatores exógenos como estilo de vida também determinam diferenças nessas transformações [1,2].

Embora na literatura se tenha registro de maior frequência de diminuição da $\mathrm{CQ}$ entre as mulheres $[2,56,77]$, entre os idosos avaliados não foi observada alteração nesta medida. Mulheres tiveram redução da CC, mas mantiveram as medidas da CQ e, como esperado, esse comportamento refletiu-se na relação cintura/quadril, que foi menor. Isto ocorreu nas faixas etárias mais jovens, enquanto entre as mais idosas as médias se mantiveram sem alteração significativa. Estudos anteriores apresentaram resultados diferentes entre si $[1,46,47,77]$, sendo importante salientar que diferenças em medidas de circunferência e razões de circunferência devem ser avaliadas com cautela pois não é possível, através destas medidas, identificar se houve perda de massa muscular ou de tecido adiposo [77].

Assim, também, houve redução da circunferência da coxa em ambos os gêneros, com raros casos citados na literatura [77]. No envelhecer de idosos saudáveis e ativos, o volume total do músculo da coxa pode sofrer uma redução de aproximadamente $20 \%$ nas mulheres e $27 \%$ nos homens, sendo o quadríceps o conjunto mais afetado. Em uma população de idosos inativos e/ou doentes, estes valores podem ser superiores [81].

Mulheres apresentam maior adiposidade subcutânea e maior perda desse tecido com o envelhecimento $[2,16]$. Isso explicaria o decréscimo da $\mathrm{CB}$ em cada faixa etária, enquanto que nos homens isso ocorreu somente entre os mais velhos [2,45-47,60,77]. Parece fundamentar, também, a redução ocorrida nas medidas das dobras cutâneas bicipital, subescapular, suprailíaca, DCT, DCA e da média de seis dobras cutâneas e AGB nas mulheres, todas estas medidas indicativas de reserva adiposa, enquanto que, entre os homens, somente ocorreu diminuição da DCA [2,47,77]. Alguns estudos, entretanto, registram diminuição da DCT tanto em homens como em mulheres [45,60,82], mas esta diminuição entre os homens parece ocorrer já na idade mais avançada.

Já a redução nas medidas da $\mathrm{AMBc}$ e da $\mathrm{CMB}$ foi observada em ambos os gêneros, assim como em outros estudos $[2,45,47,60]$. Tanto homens como mulheres estão sujeitos à perda de massa muscular no envelhecimento, mas esta parece ser mais tardia entre os homens. A CP, bastante referida como sinalizador de reserva de massa muscular, sofreu redução entre as mulheres mas manteve-se inalterada entre os homens, comportamento já referido em estudos prévios $[56,77]$, embora haja registro de perda também entre os homens $[45,60]$.

As medidas da circunferência do punho, das dobras cutâneas da coxa, panturrilha e peitoral, a RCE, a relação cintura/panturrilha e o índice de conicidade não apresentaram modificações em relação às faixas etárias. Entretanto, não foi possível comparar estes resultados com a literatura, pois são escassos os dados sobre o comportamento dessas medidas no envelhecimento [77]. Medidas como a dobra cutânea peitoral 
e da coxa têm sido utilizadas em equações preditivas de gordura corporal [25], mas pouco estudadas isoladamente Os resultados deste estudo, portanto, podem, no futuro, auxiliar na discussão de novos achados.

$\mathrm{Na}$ associação das medidas antropométricas com fragilidade, observa-se que raros estudos têm se dedicado a este assunto. O que se vê na literatura é a associação do estado nutricional, magreza e obesidade, aspectos importantes no curso molecular, fisiológico e clínico da fragilidade [8]. Mesmo sem a possibilidade de comparação das diversas medidas antropométricas avaliadas em nosso estudo, pela falta de estudos similares na literatura, o comportamento dessas medidas em relação aos estágios de fragilidade permite que se possa associar as características das medidas aos conceitos da síndrome fragilidade.

A diminuição da estatura e da altura do joelho (medidas de compleição física), a cada estágio de fragilidade, está congruente com a conceituação de fragilidade como um distúrbio de desperdício, ou seja, um estado de diminuição de reservas e declínio cumulativo de diversos sistemas fisiológicos [4,83]. Sabe-se, também, que o decréscimo da massa e da força muscular contribui significativamente para vulnerabilidade ao stress, que se manifesta na fragilidade [4]. A medida da CP, considerada medida sensível às mudanças de massa livre de gordura que ocorrem no envelhecimento e na inatividade, foi menor entre os frágeis em relação aos pré-frágeis e robustos.

Avaliando idosos da comunidade, Landi et al. [12] sugeriram que a CP aumentada está associada com menor nível de fragilidade e melhor desempenho funcional. A massa muscular está fortemente implicada na manutenção da independência durante o envelhecimento. A CP é considerada um potencial marcador de função física, uma ferramenta barata, simples e não invasiva, valiosa na saúde pública e nas decisões clínicas [12]. Faz parte, também, da Mini Avaliação Nutricional, instrumento bastante utilizado para rastreio de risco nutricional em idosos [84].

Assim, também, a $\mathrm{CMB}$ e a $\mathrm{AMBc}$, medidas representativas de reserva muscular, foram menores nos frágeis em relação aos robustos. Perda de massa muscular pode levar à sarcopenia, uma das principais características fisiopatológicas da fragilidade. Sarcopenia e fragilidade se sobrepõem; a maioria dos idosos frágeis apresentam sarcopenia e idosos com sarcopenia são também frágeis [9]. Segundo o consenso de fragilidade, a sarcopenia, bem como o declínio funcional, podem ser fatores de risco para a fragilidade, como, também, podem ser decorrentes de um estado de fragilidade instalado por outras condições de saúde [14]. Enoki et al. [85] afirmam que CMB e AMBc, além de indicadores de massa muscular são, também, indicadores de desnutrição e podem ser uma forma prática e adequada para identificar vulnerabilidade de idosos da comunidade [85].

$\mathrm{A}$ medida da $\mathrm{CAB}$ foi menor nos frágeis em relação aos pré-frágeis e robustos, compatível com achados sobre sua redução com o envelhecimento [61] e estudo sobre sarcopenia em idosos, que verificou uma $\mathrm{CAB}$ menor entre idosos sarcopênicos [86]. A perda de massa magra, entretanto, nem sempre é acompanhada de perda de massa gorda e, muitas vezes, até, há um aumento do tecido adiposo. Esse estado é denominado obesidade sarcopênica [9] e os resultados aqui encontrados auxiliam na identificação dessa condição nos idosos. Tanto as medidas indicativas de reserva adiposa (dobras cutâneas bicipital, da panturrilha, da coxa, peitoral, DCT e AGB) como medidas representativas da distribuição da gordura corporal (RCE, índice sagital, relação DAS/Estatura, relação cintura/coxa, relação cintura/ panturrilha e índice de conicidade) foram superiores nos idosos frágeis. Adiposidade abdominal, também, pode ser particularmente importante na patogênese da fragilidade, pois está associada à inflamação sistêmica, mediando a sua ligação com síndrome metabólica [87]. Além disso, aqueles com a CC aumentada têm maiores marcadores de estresse oxidativo [88]. O impacto adicional da obesidade abdominal na fragilidade em idosos reforça a importância da dieta adequada e do exercício físico nessa população. Mesmo entre idosos abaixo do peso, aqueles com uma maior CC têm mais probabilidade de serem frágeis [89].

O uso de técnicas diretas de avaliação da composição corporal tem sido sugerido como ideal para detectar mudanças durante o envelhecimento, como as relacionadas à sarcopenia e à fragilidade. Em determinadas condições, como na atenção básica, isto não é possível. Assim, os resultados aqui encontrados, juntamente com informações prévias na literatura, permitem sugerir que muitas medidas antropométricas podem ser utilizadas para indicar situações de vulnerabilidade dos idosos.

Existem poucas informações disponíveis sobre medidas antropométricas de idosos, especialmente em países em desenvolvimento. Estudos são necessários para que sejam determinadas medidas de referência para dados antropométricos de idosos e pontos de corte adequados para esta faixa etária [90]. No Brasil, são raros os estudos populacionais multidimensionais avaliando o perfil antropométrico de idosos [2] e, no 
estado do Rio Grande do Sul, o EMI-SUS é pioneiro na pesquisa deste assunto em idosos da atenção básica [17], que é o primeiro nível de atendimento do sistema de saúde público [91]. O conjunto abrangente de medidas avaliado neste estudo é, também, uma conduta pioneira, pela inclusão de medidas ainda pouco estudadas por outros autores e com raros registros bibliográficos. Esta característica propicia dados que poderão ser comparados futuramente.

Estes achados estão sujeitos às mesmas limitações dos estudos transversais prévios no que se refere à interpretação das diferenças ocorridas em cada faixa etária. Estudos transversais não permitem observar as relações temporais entre as alterações ocorridas nas medidas antropométricas no curso da fragilidade e no tempo. Também é necessário ter cautela na extrapolação dos resultados, pois esta amostra foi selecionada e é constituída de idosos da comunidade, atendidos na atenção básica, a maioria de origem caucasiana.

Concluindo, no presente estudo observou-se que, em idosos assistidos na atenção básica de saúde, as medidas antropométricas mostraram-se associadas com gênero, idade e fragilidade: as médias das medidas de tamanho e compleição e das medidas relacionadas às reservas de massa muscular foram maiores entre os homens; a média das medidas indicativas de reserva adiposa foi maior nas mulheres; as diferenças das medidas antropométricas entre as faixas etárias foram mais frequentes nas mulheres; idosos frágeis apresentaram medidas inferiores de tamanho e compleição; fragilidade mostrou-se associada com perda de massa muscular avaliada pelas medidas antropométricas empregadas.

A constatação de que várias medidas antropométricas têm sido pouco avaliadas em idosos e os resultados aqui encontrados, relacionando diversas medidas a condições de saúde nesta população, estimula a busca por maiores informações sobre o assunto, pois a antropometria, dentro de sua simplicidade e fácil aplicação, pode ser uma ferramenta muito útil na avaliação de idosos, principalmente no nível de atenção básica.

\section{REFERÊNCIAS}

1. Perissinotto E, Pisent C, Sergi G, Grigoletto F, Enzi G. Anthropometric measurements in the elderly: age and gender differences. Br J Nutr. 2002;87(02):177-86. http://dx.doi.org/10.1079/BJN2001487

2. Barbosa AR, Souza JMP, Lebrao ML, Laurenti R, Marucci MdFN. Anthropometry of elderly residents in the city of São Paulo, Brazil. Cad Saude Publica. 2005;21(6):1929-38. http://dx.doi.org/10.1590/S0102-311X2005000600043

3. Woo J. Nutritional strategies for successful aging. Med Clin North Am. 2011;95(3):477-93. http://dx.doi.org/10.1016/j.mcna.2011.02.009

4. Fried LP, Tangen CM, Walston J, Newman AB, Hirsch C, Gottdiener J, Seeman T, Tracy R, Kop WJ, Burke G, McBurnie MA. Frailty in older adults: evidence for a phenotype. J Gerontol. 2001;56A(3):M146-M56. http://dx.doi.org/10.1093/gerona/56.3.M146

5. Bergman H, Ferrucci L, Guralnik J, Hogan DB, Hummel S, Karunananthan S, Wolfson C. Frailty: An emerging research and clinical paradigm - issues and controversies. J Gerontol A Biol Sci Med Sci. 2007;62(7):731-7. http://dx.doi.org/10.1093/gerona/62.7.731

6. Kuzuya M. Process of physical disability among older adults -contribution of frailty in the super-aged society. Nagoya J Med Sci. 2012;74:31-7.

7. Landi F, Russo A, Liperoti R, Pahor M, Tosato M, Capoluongo E, Bernabei R, Onder G. Midarm muscle circumference, physical performance and mortality: results from the aging and longevity study in the Sirente geographic area (ilSIRENTE study). Clinical Nutrition. 2010;29(4):441-7. http://dx.doi.org/10.1016/j.clnu.2009.12.006

8. Walston J, Hadley EC, Ferrucci L, Guralnik JM, Newman AB, Studenski SA, Ershler WB, Harris T, Fried L. Research agenda for frailty in older adults: toward a better understanding of physiology and etiology: summary from the American Geriatrics Society/National Institute on Aging Research Conference on frailty in older adults. J Am Geriatr Soc. 2006;54(6):991-1001. http://dx.doi.org/10.1111/j.15325415.2006.00745.x

9. Cruz-Jentoft AJ, Baeyens JP, Bauer JM, Boirie Y, Cederholm T, Landi F, Martin FC, Michel JP, Rolland Y, Schneider SM, Topinková E, Vanderwoude M, Zamboni M. Sarcopenia: European consensus on definition and diagnosis: report of the European Working Group on Sarcopenia in Older People. Age Ageing. 2010;39(4):412-23. http://dx.doi.org/10.1093/ageing/afq034

10. Brasil. Ministério da Saúde. Caderno de atenção básica 19 - Envelhecimento e saúde da pessoa idosa.Brasília (DF): Ministério da Saúde; 2009. p. 36.

11. WHO. World Health Organization. Physical status: the use and interpretation of anthropometry. Report of a WHO Expert Committee. Geneva: Worl Health Organization, 1995.

12. Landi F, Onder G, Russo A, Liperoti R, Tosato M, Martone AM, Capoluongo E, Barnabei R. Calf circumference, frailty and physical performance among older adults living in the community. Clinical Nutrition. 2014;33(3):539-44. http://dx.doi.org/10.1016/j.clnu.2013.07.013

13. Seidell J, Visscher TLS. Body weight and weight change and their health implications for the elderly. Eur J Clin Nutr. 2000;54(3):S33-S9. http://dx.doi.org/10.1038/sj.ejcn.1601023 
14. Morley JE, Vellas B, van Kan GA, Anker SD, Bauer JM, Bernabei R, Cesari M, Chumlea WC, Doehner W, Evans J, Fried LP, Guralnik JM, Katz PR, Malmstrom TK, McCarter RJ, Robledo LMG, Rockwood K, von Haehling S, Vandewoude MF, Walston J. Frailty consensus: a call to action. JAMDA. 2013;14(6):392-7. http://dx.doi.org/10.1016/j.jamda.2013.03.022

15. Bós AJG. Características fisiológicas do processo do envelhecimento. In: Busnello FM, editor. Aspectos nutricionais do processo do envelhecimento. São Paulo: Editora Atheneu; 2007.

16. Delarue J, Constans T, Malvy D, Pradignac A, Couet C, Lamisse F. Anthropometric values in an elderly French population. Br J Nutr. 1994;71:295-302. http://dx.doi.org/10.1079/BJN19940135

17. Gomes I, Nogueira EL, Engroff P, Ely LS, Schwanke CHA, De Carli GA, Resende TL. The multidimensional study of the elderly in the family health strategy in Porto Alegre, Brazil (EMI-SUS). PAJAR. 2013;1(1):20-4.

18. Marfell-Jones M, Olds T, Stewart A, Carter L. International standars for anthropometric assessment. North-West University. South Africa.: International Society for the Advancement of Kinanthropometry. School of Biokinetics, Recreation and Sport.; 2006.

19. NHANES. Anthropometry procedures manual. Center of Disease Control; 2007 [Cited 2015 Mar 29]. Available from: http://www.cdc. gov/nchs/data/nhanes/nhanes_07_08/manual_an.pdf

20. Garrow JS, Webster J. Quetelet's index (W/H2) as a measure of fatness. Int J Obes. 1985;9(2):147-53.

21. Chumlea WC, Roche AF, Steinbaugh ML. Estimating stature from knee height for persons 60 to 90 years of age. J Am Geriatr Soc. 1985;33(2):116-20. http://dx.doi.org/10.1111/j.1532-5415.1985.tb02276.x

22. Frisancho AR. New norms of upper limb fat and muscle areas for assessment of nutritional status. Am J Clin Nutr. 1981;34:2540-5.

23. Lohman TG, Roche AF, Martorell R. Anthropometric standardization reference manual. Champaign: Human Kinetics Books.; 1988.

24. Janssen I, Katzmarzyk PT, Ross R. Body mass index, waist circunference, and health risk. evidence in support of current National Institutes of Health guidelines. Arch Intern Med. 2002;162:2074-9. http://dx.doi.org/10.1001/archinte.162.18.2074

25. Jackson AS, Pollock ML. Pratical assessment of body composition. Phys Sportsmed. 1985;13:256-62.

26. Coelho AC, Fausto MA. Avaliação pelo nutricionista. In: Maciel A. Avaliação multidisciplinar do paciente geriátrico. Rio de Janeiro: Revinter; 2002. p. 121-56.

27. Chumlea WC, Steinbaugh ML, Roche AF, Mukherjee D, Gopalaswamy N. Nutritional assessment of the elderly through anthropometry. Columbus: Ross Laboratories; 1984.

28. Chumlea WC, Guo S, Roche AF, Steinbaugh ML. Prediction of body weight for the nonambulatory elderly from anthropometry. J Am Diet Assoc. 1988;88(5):564-8.

29. Tyagi R, Kapoor S, Kapoor AK. Body composition and fat distribution pattern of urban elderly females, Delhi, India. Coll Antropol. $2005 ; 29(2): 493-8$

30. Richelsen B, Pedersen SB. Associations between different anthropometric measurements of fatness and metabolic risk parameters in non-obese, healthy, middle-aged men. Int J Obes Relat Metab Disord. 1995;19(3):169-74.

31. Ohrvall M, Berglund L, Vessby B. Sagittal abdominal diameter compared with other anthropometric measurements in relation to cardiovascular risk. Int J Obes. 2000;24:497-501. http://dx.doi.org/10.1038/sj.ijo.0801186

32. Risérus U, Arnlov J, Brismar K, Zethelius B, Berglund L, Vessby B. Sagittal abdominal diameter is a strong anthropometric marker of insulin resistance and hyperproinsulinemia in obese men. Diabetes Care. 2004;27:2041-6. http://dx.doi.org/10.2337/diacare.27.8.2041

33. Kamimura MA, Baxmann A, Sampaio LR, Cuppari L. Avaliação nutricional. In: Cuppari L. Nutrição: nutrição clínica no adulto. $2^{\mathfrak{a}}$ ed. Barueri - São Paulo: Manole; 2005.

34. Heymsfield SB, McManus C, Smith J, Stevens V, Nixon DW. Anthropometric measurement of muscle mass - revised equations for calculating bone-free arm muscle area. Am J Clin Nutr. 1982;36:680-90.

35. Vasques ACJ, Rosado LEFPL, Rosado GP, Ribeiro RCL, Franceschini SCC, Geloneze B, Priore SE, Oliveira DR. Habilidade de indicadores antropométricos e de composição corporal em identificar a resistência à insulina. Arq Bras Endocrinol Metab. 2009;53(1):72-9. http:// dx.doi.org/10.1590/S0004-27302009000100011

36. Carlsson AC, Riserus U, Engstrom G, Arnlov J, Melander O, Leander K, Gigante B, Hellénius ML, Faire U. Novel and established anthropometric measures and the prediction of incident cardiovascular disease: a cohort study. Int J Obes. 2013;37(12):1579-85. http:/ dx.doi.org/10.1038/ijo.2013.46

37. Gavi S, Feiner JJ, Melendez MM, Mynarcik DC, Gelato MC, Mc.Nurlan MA. Limb fat to trunk fat ratio in elderly persons is a strong determinant of insulin resistance and adiponectin levels. J Gerontol Med Sci. 2007;62(9):997-1001. http://dx.doi.org/10.1093/ gerona/62.9.997

38. Valdez R. A simple model-based index of abdominal adiposity. J Clin Epidemiol. 1991;46(5):491-4. http://dx.doi.org/10.1016/08954356(91)90059-i

39. Fess EE. Grip strength. In: Casanova JS. Clinical assessment recommendations. Chicago: American Society of Hand Therapists; 1992. p. 41-5.

40. Batistoni SST, Neri AL, Cupertino APFB. Validity of the Center for Epidemiological Studies Depression Scale among Brazilian elderly. Rev Saude Publica. 2007;41(4):1-7.

41. Taylor HL, Jacobs DR, Jr., Schuker B, Knudsen J, Leon AS, Debacker G. A questionnaire for the assessment of leisure-time physical activities. J Chronic Dis. 1978;31:745-55. http://dx.doi.org/10.1016/0021-9681(78)90058-9

42. Tresignie J, Scafoglieri A, Pieter Clarys J, Cattrysse E. Reliability of standard circumferences in domain-related constitutional applications. Am J Hum Biol. 2013;25(5):637-42. http://dx.doi.org/10.1002/ajhb.22423 
43. Lipschitz DA. Screening for nutritional status in the elderly. Prim Care. 1994;21(1):55-67.

44. Mastroeni MF, Mastroeni SSdBS, Erzinger GS, Marucci MdFN. Antropometria de idosos residentes no município de Joinville-SC, Brasil. Rev Bras Geriatr Gerontol. 2010;13(1):29-40. http://dx.doi.org/10.1590/S1809-98232010000100004

45. Coqueiro RdS, Barbosa AR, Borgatto AF. Anthropometric measurements in the elderly of Havana, Cuba: Age and sex differences. Nutrition. 2009;25(1):33-9. http://dx.doi.org/10.1016/j.nut.2008.07.007

46. Canaan Rezende FA, Queiroz Ribeiro A, Priore SE, Castro Franceschini SD. Anthropometric differences related to genders and age in the elderly. Nutr Hosp. 2015;32(02):757-64

47. Santos DM, Sichieri R. Body mass index and measures of adiposity among elderly adults. Rev Saude Publica. 2005;39(2):163-8.

48. Velázquez-Alva MC, Castillo-Martinez L, Irigoyen-Camacho E, Zepeda-Zepeda MA, Gutiérrez-Robledo LM, Cisneros-Moysen P. Estudio antropométrico en un grupo de hombres y mujeres de la tercera edad en la Ciudad de Mexico. Salud Publica Mex. 1996;38(6):466-74.

49. Velázquez-Alva MC, Irigoyen ME, Zepeda M, Sanchez VM, Garcia Cisneros MP, Castillo LM. Anthropometric measurements of a sixtyyear and older Mexican urban group. J Nutr Health Aging. 2004;8(5):350-4.

50. Pfrimer K, Ferriolli E. Avaliação nutricional do idoso. In: Vitolo MR. Nutrição da gestação ao envelhecimento. Rio de Janeiro: Editora Rubio; 2008. p.436-49.

51. Fontanive R, Paula TP, Peres WAF. Avaliação da composição corporal de adultos. In: Duarte ACG. Avaliação nutricional: aspectos clínicos e laboratoriais. São Paulo: Atheneu. 2007. p. 41-63.

52. Durnin JVGA, Womersley J. Body fat assessed from total body density and its estimation from skinfold thickness: measurements on 481 men and women aged from 16 to 72 years. Br J Nutr. 1974;32:77-97. http://dx.doi.org/10.1079/BJN19740060

53. Kuczmarski MF, Kuczmarski RJ, Najjar M. Descriptive anthropometric reference data for older americans. J Am Diet Assoc. 2000;100(1): 59-66. http://dx.doi.org/10.1016/S0002-8223(00)00021-3

54. Nazare JA, Smith J, Borel AL, Aschner P, Barter P, Van Gaal L, Tan CE, Wittchen HU, Matsuzawa Y, Kadowaki T, Ross R, BrulleWohlhueter C, Alméras N, Haffner SM, Balkau B, Després JP. Usefulness of measuring both body mass index and waist circumference for the estimation of visceral adiposity and related cardiometabolic risk profile (from the INSPIRE ME IAA study). Am J Cardiol. 2015;115(3):307-15. http://dx.doi.org/10.1016/j.amjcard.2014.10.039

55. Smith U. Abdominal obesity: a marker of ectopic fat accumulation. J Clin Invest. 2015;125(5):1790-2. http://dx.doi.org/10.1172/JCI81507

56. Sánchez-García S, García-Pe-a C, Duque-López M, Juárez-Cedillo T, Cortés-Nú-ez A, Reyes-Beaman S. Anthropometric measures and nutritional status in a healthy elderly population. BMC Public Health. 2007;7(1):2. http://dx.doi.org/10.1186/1471-2458-7-2

57. Cameron AJ, Magliano DJ, Shaw JE, Zimmet PZ, Carstensen B, Alberti KGMM, Tuomilehto J, Barr ELM, Pauvaday VK, Kowlessur $\mathrm{S}$, Söderberg S. The influence of hip circumference on the relationship between abdominal obesity and mortality. Int J Epidemiol. 2012;41(2):484-94. http://dx.doi.org/10.1093/ije/dyr198

58. Manolopoulos KN, Karpe F, Frayn KN. Gluteofemoral body fat as a determinant of metabolic health. Int J Obes. 2010;34(6):949-59. http://dx.doi.org/10.1038/ijo.2009.286

59. Snijder MB, Dekker JM, Visser M, Bouter LM, Stehouwer CD, Kostense PJ, Yudkin J, Heine RJ, Nijpels G, Seidell J. Associations of hip and thigh circumferences independent of waist circumference with the incidence of type 2 diabetes: the Hoorn Study. Am J Clin Nut. 2003;77:1192-7.

60. Santos JL, Albala C, Lera L, Garcia C, Arroyo P, Perez-Bravo F, Pérez-Bravo F, Angel B, Peláez M. Anthropometric measurements in the elderly population of Santiago, Chile. Nutrition. 2004;20(5):452-7. http://dx.doi.org/10.1016/j.nut.2004.01.010

61. Anakwe RE, Huntley JS, McEachan JE. Grip strength and forearm circumference in a healthy population. J Hand Surg Eur Vol. 2007;32(2):203-9. http://dx.doi.org/10.1016/j.jhsb.2006.11.003

62. Chagas P, Caramori P, Barcellos C, Galdino TP, Gomes I, Schwanke CHA. Associação de diferentes medidas e índices antropométricos com a carga aterosclerótica coronariana. Arq Bras Cardiol. 2011;97(5):397-401. http://dx.doi.org/10.1590/S0066-782X2011005000093

63. Ben-Noun L, Laorf A. Relationship of neck circunference to cardiovascular risc factors. Obes Res. 2003;11(2):226-31. http://dx.doi. org/10.1038/oby.2003.35

64. Subramanian S, Jayaraman G, Majid H, Aguilar R, Surani S. Influence of gender and anthropometric measures on severity of obstructive sleep apnea. Sleep Breath. 2012;16(4):1091-5. http://dx.doi.org/10.1007/s11325-011-0607-9

65. Vasques AC, Rosado L, Rosado G, Ribeiro RdC, Franceschini S, Geloneze B. Indicadores antropométricos de resistência à insulina. Arq Bras Cardiol. 2010;95(1):e14-e23. http://dx.doi.org/10.1590/S0066-782X2010001100025

66. Martinez-Rivera C, Abad J, Fiz JA, Rios J, Morera J. Usefulness of truncal obesity indices as predictive factors for obstructive sleep apnea syndrome. Obesity. 2008;16(1):113-8. http://dx.doi.org/10.1038/oby.2007.20

67. Smith DA, Ness EM, Herbert R, Schechter CB, Phillips RA, Diamond JA, Landrigan PJ. Abdominal diameter index: a more powerful anthropometric measure for prevalent coronary heart disease risk in adult males. Diabetes Obes Metb. 2005;7(4):370-80. http://dx.doi. $\operatorname{org} / 10.1111 / \mathrm{j} .1463-1326.2004 .00406 . x$

68. Gobbo LA, Dourado DAQS, Almeida MFd, Duarte YAdO, Lebrão ML, Marucci MDFN. Massa muscular de idosos do município de São Paulo - Estudo SABE: Saúde, Bem-estar e Envelhecimento. DOI: 10.5007/1980-0037.2012v14n1p1. Rev Bras Cineantrop Desemp Hum. 2012;14(1). http://dx.doi.org/10.5007/1980-0037.2012v14n1p1

69. Ashwell M, Hsieh SD. Six reasons why the waist-to-height ratio is a rapid and effective global indicator for health risks of obesity and how its use could simplify the international public health message on obesity. Int J Food Sci Nutr. 2005;56(5):303-7. http://dx.doi. org/10.1080/09637480500195066 
70. Pitanga FJG, Lessa I. Razão cintura-estatura como discriminador do risco coronariano de adultos. Rev Assoc Med Bras. 2006;52(3): 157-61. http://dx.doi.org/10.1590/S0104-42302006000300016

71. Mukuddem-Petersen J, Snijder MB, Van Dam RM, Dekker JM, Bouter LM, Stehouwer CDA, Heine RJ, Nijpels G, Seidell J. Sagittal abdominal diameter: no advantage compared with other anthropometric measures as a correlate of components of the metabolic syndrome in elderly from the Hoorn Study. Am J Clin Nutr. 2006;84:995-1002.

72. Sampaio LR, Simões Ej, Assis AMO, Ramos LR. Validity and reliability of the sagittal abdominal diameter as a predictor of visceral abdominal fat. Arq Bras Endocrinol Metabol. 2007;51(6):980-6. http://dx.doi.org/10.1590/S0004-27302007000600013

73. Vasques AC, Cassani RS, Forti AC, Vilela BS, Pareja JC, Tambascia MA, Geloneze B. Sagittal abdominal diameter as a surrogate marker of insulin resistance in an admixtured population-brazilian metabolic syndrome study (BRAMS). PloS One. 2015;10(5):e0125365. http:// dx.doi.org/10.1371/journal.pone.0125365

74. Madden AM, Smith S. Body composition and morphological assessment of nutritional status in adults: a review of anthropometric variables. J Hum Nutr Diet. 2014:00:00-00. http://dx.doi.org/10.1111/jhn.12278

75. Kahn HS, Rissanen H, Bullard KM, Knekt P. The population distribution of the sagittal abdominal diameter (SAD) and SAD/height ratio among Finnish adults. Clin Obes. 2014;4(6):333-41. http://dx.doi.org/10.1111/cob.12078

76. Klee Oehlschlaeger MH, Alberici Pastore C, Schuler Cavalli A, Gonzalez MC. Nutritional status, muscle mass and strength of elderly in southern Brazil. Nutr Hosp. 2014;31(01):363-70.

77. Baumgartner RN, Stauber PM, McHugh D, Koehler KM, Garry PJ. Cross-sectional age differences in body composition in persons $60+$ years of age. J Gerontol. 1995;50A(6):M307-M16. http://dx.doi.org/10.1093/gerona/50A.6.M307

78. Coin A, Perissinotto E, Enzi G, Zamboni M, Inelmen EM, Frigo AC, Manzato E, Busetto L, Buja A, Sergi G. Predictors of low bone mineral density in the elderly: the role of dietary intake, nutritional status and sarcopenia. Eur J Clin Nutr. 2008;62(6):802-9. http://dx.doi. org/10.1038/sj.ejcn.1602779

79. Alswat K, Adler SM. Gender differences in osteoporosis screening: retrospective analysis. Arch Osteoporos. 2012;7:311-3. http://dx.doi. org/10.1007/s11657-012-0113-0

80. Closs VE, Feoli AMP, Schwanke CHA. Altura do joelho como medida alternativa confiável na avaliação nutricional de idosos. Rev Nutr 2015;28(5):475-84. http://dx.doi.org/10.1590/1415-52732015000500002

81. Maden-Wilkinson TM, McPhee JS, Rittweger J, Jones DA, Degens H. Thigh muscle volume in relation to age, sex and femur volume. Age (Dordr). 2014;36(1):383-93. http://dx.doi.org/10.1007/s11357-013-9571-6

82. Barbosa AR, Souza JMP, Lebrao ML, Marucci MF. Relação entre estado nutricional e força de preensão manual em idosos do município de São Paulo, Brasil. Rev Bras Cineantrop Desemp Hum. 2006;8(8):8.

83. Rolland Y, Czerwinski S, Abellan Van Kan G, Morley JE, Cesari M, Onder G, Woo J, Baumgartner R, Pillard F, Boirie Y, Chumlea WMC, Vellas B. Sarcopenia: its assessment, etiology, pathogenesis, consequences and future perspectives. J Nutr Health Aging. 2008;12(7): 433-50. http://dx.doi.org/10.1007/BF02982704

84. Vellas B, Garry PJ, Guigoz Y. Mini Nutritional Assessment (MNA): research and practice in the elderly. Switzerlan: Nestlé Nutrition Workshop Series. Clinical \& Performance Programme: 1999.

85. Enoki H, Kuzuya M, Masuda Y, Hirakawa Y, Iwata M, Hasegawa J, Izawa S, Iguchi A. Anthropometric measurements of mid-upper arm as a mortality predictor for community-dwelling Japanese elderly: the Nagoya Longitudinal Study of Frail Elderly (NLS-FE). Clinical Nutrition. 2007;26(5):597-604. http://dx.doi.org/10.1016/j.clnu.2007.06.008

86. Campbell TM, Vallis LA. Predicting fat-free mass index and sarcopenia in assisted-living older adults. Age (Dordr). 2014;36(4):9674 http://dx.doi.org/10.1007/s11357-014-9674-8

87. Zuliani G, Volpato S, Galvani M, Ble A, Bandinelli S, Corsi AM, Lauretani F, Maggio M, Guralnik JM, Fellin R, Ferrucci L. Elevated C-reactive protein levels and metabolic syndrome in the elderly: The role of central obesity data from the InChianti study. Atherosclerosis. 2009;203(2):626-32. http://dx.doi.org/10.1016/j.atherosclerosis.2008.07.038

88. Porter Starr KN, McDonald SR, Bales CW. Obesity and physical frailty in older adults: a scoping review of lifestyle intervention trials. J Am Med Dir Association. 2014;15(4):240-50. http://dx.doi.org/10.1016/j.jamda.2013.11.008

89. Hubbard RE, Lang IA, Llewellyn DJ, Rockwood K. Frailty, body mass index, and abdominal obesity in older people. J Gerontol A Biol Sci Med Sci. 2010;65(4):377-81. http://dx.doi.org/10.1093/gerona/glp186

90. Cervi A, Franceschini SdCC, Priore SE. Análise crítica do uso do índice de massa corporal para idosos. Rev Nutr. 2005;18(6):765-75. http://dx.doi.org/10.1590/S1415-52732005000600007

91. Macinko J, MHarris MJ, Phil D. Brazil's Family Health Strategy - delivering community-based primary care in a universal health system. N Engl J Med. 2015;372(23):2177-81. http://dx.doi.org/10.1056/NEJMp1501140 C 